\title{
WestVirginiaUniversity
}

THE RESEARCH REPOSITORY @ WVU

Graduate Theses, Dissertations, and Problem Reports

2012

\section{Optimal Portfolio and Consumption with Transaction Costs}

\author{
Zheng Zhang \\ West Virginia University
}

Follow this and additional works at: https://researchrepository.wvu.edu/etd

\section{Recommended Citation}

Zhang, Zheng, "Optimal Portfolio and Consumption with Transaction Costs" (2012). Graduate Theses, Dissertations, and Problem Reports. 3572.

https://researchrepository.wvu.edu/etd/3572

This Dissertation is protected by copyright and/or related rights. It has been brought to you by the The Research Repository @WVU with permission from the rights-holder(s). You are free to use this Dissertation in any way that is permitted by the copyright and related rights legislation that applies to your use. For other uses you must obtain permission from the rights-holder(s) directly, unless additional rights are indicated by a Creative Commons license in the record and/ or on the work itself. This Dissertation has been accepted for inclusion in WVU Graduate Theses, Dissertations, and Problem Reports collection by an authorized administrator of The Research Repository @ WVU.

For more information, please contact researchrepository@mail.wvu.edu. 


\title{
Optimal Portfolio and Consumption with Transaction Costs
}

\author{
Zheng Zhang \\ Dissertation submitted to the \\ Eberly College of Arts and Sciences \\ at West Virginia University \\ in partial fullment of the requirements \\ for the degree of \\ Doctor of Philosophy \\ in \\ Mathematics
Harvey Diamond, Ph.D.
Alexander Kurov, Ph.D.
Dening Li, Ph.D. \\ Harumi Hattori, Ph.D., Chair \\ Sherman Riemenschneider, Ph.D. \\ Department of Mathematics \\ Morgantown, West Virginia \\ 2012
}

Keywords: Hamilton-Jacobi-Bellman equation; Transaction cost;

Explicit solutions; Verification theorem; Viscosity solutions

Copyright 2012 Zheng Zhang 


\section{Abstract \\ Optimal Portfolio and Consumption with Transaction Costs}

\section{Zheng Zhang}

In Chapter 1, we study optimal portfolio and consumption with both fixed and proportional transaction costs. For a power utility function we find an explicit solution to the HJB equation governing the no-transaction region. Based on the explicit solution, we formulate a combined stochastic and impulse control problem as a quasi-variational inequality and find the transaction regions, the no-transaction region, and the boundary curves separating them. We show that the explicit solution we find satisfies the verification theorem and it is also a viscosity solution for the quasi-variational inequality. We present numerical results where we compare the various cases of the fixed and proportional transaction costs.

In Chapter 2, we discuss the optimal portfolio and consumption on multiple risky assets with both fixed and proportional transaction costs. Explicit solutions to the corresponding HJB equations are provided. The explicit solutions are viscosity solutions. Numerical results for two risky assets and $N$ risky assets are given. 


\section{Acknowlegements}

First, I'd like to express my sincere thanks to my advisor, Dr. Harumi Hattori, for his guidance, encouragement, help and support during all these years. It has been a great pleasure to work under his supervision and I am lucky enough to have had a chance to learn from him. What I learned from him will benefit me all my life.

Secondly, I would like to thank my committee members, Professors Harvey Diamond, Alexander Kurov, Dening Li and Sherman Riemenschneider. Their advices throught the process have been invaluable.

Thirdly, I would like to thank my parents, my husband and daughter for their everlasting support without which this work could not have come out.

Finally, I would like to thank the Department of Mathematics and Eberly College of Arts and Sciences at West Virginia University for providing me an excellent study environment and support during my study as a graduate student. 


\section{Contents}

1 One Risky Asset Problem 1

1.1 Introduction . . . . . . . . . . . . . . . . 1

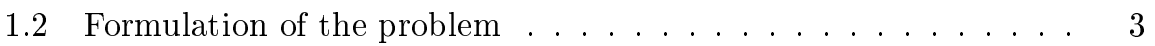

1.3 Solutions to HJB equation . . . . . . . . . . . . . 5

1.4 Analytical study of the boundaries between transaction regions

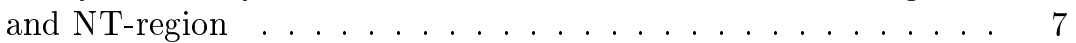

1.5 Verification Theorem . . . . . . . . . . . . . . . . . . 12

1.6 Viscosity Solutions . . . . . . . . . . . . . . . . . . . . 19

1.7 Numerical results . . . . . . . . . . . . . . . . . . . . . . . 22

1.7.1 The case when $\gamma>m \ldots \ldots \ldots \ldots 23$

1.7.1.1 The case when $k>0, \lambda>0 . \ldots \ldots 23$

1.7.1.2 The case when $k>0, \lambda=0 . \ldots \ldots \ldots 24$

1.7.1.3 The cases when $k=0 . \ldots \ldots \ldots$

1.7.2 The case when $m>\gamma \ldots \ldots \ldots \ldots$

1.7.3 The approximate transaction boundaries . . . . . . . 27

1.8 Explicit solutions for exponential and natural logarithm utility functions . . . . . . . . . . . . . . . . . 29

1.8.1 Exponential utility function . . . . . . . . . . . . 29

1.8.2 Natural Logarithm utility function . . . . . . . . . . . 31

2 Multiple Risky Assets Problem 34

2.1 Introduction . . . . . . . . . . . . . . . . . . 34

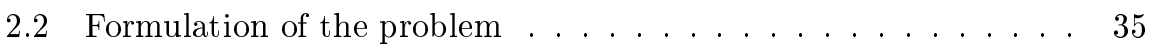

2.3 Solutions to HJB equation . . . . . . . . . . . . . 37

2.4 Viscosity Solution . . . . . . . . . . . . . . . . . . 39

2.5 Numerical results . . . . . . . . . . . . . . . . . . 44

2.5 .1 Two risky assets . . . . . . . . . . . . . 44

2.5.1.1 The case when $k=0 . \ldots \ldots \ldots . \ldots 44$

2.5.1.2 The case when $k>0 . \ldots \ldots \ldots . \ldots 45$

$2.5 .2 \quad N$ risky assets . . . . . . . . . . . . . . 48

2.5.2.1 Algorithm I . . . . . . . . . . . . . . . 48

2.5.2.2 Algorithm II . . . . . . . . . . . . . . 50 


\section{List of Figures}

1.1 Graphs for several curves. . . . . . . . . . . . . . 8

1.2 Trading details . . . . . . . . . . . . . . . 17

1.3 (Left) The value function $v(x, y)=a x^{\gamma}+\frac{y^{m}}{m}$. (Right) The contour curves for the value function $v(x, y)=a x^{\gamma}+\frac{y^{m}}{m}$. . . . . 23

1.4 Transaction regions and NT region when $k>0, \lambda>0$ and

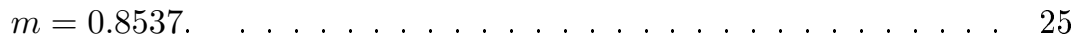

1.5 Transaction regions and NT region when $k>0, \lambda=0$ and

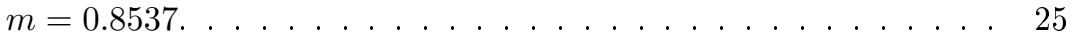

1.6 Transaction regions and NT region when $k=0, \lambda=0, m=$ $0.8537 \ldots \ldots \ldots \ldots \ldots \ldots \ldots \ldots$

1.7 Transaction regions and NT region when $k=0, \lambda>0, m=0.8537 .27$

1.8 Transaction regions and NT region when $k>0, \lambda=0$ and

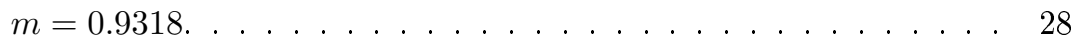

1.9 Transaction regions and NT region when $k>0, \lambda>0$ and

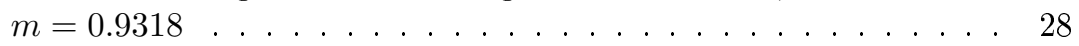

1.10 Transaction regions and NT region when $k=0.1, \lambda>0$ and $m=0.9318 \ldots \ldots \ldots \ldots \ldots \ldots \ldots \ldots$

1.11 Transaction regions and NT region when $k=0.3, \lambda>0$ and

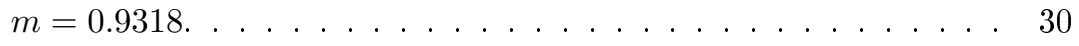

1.12 Transaction regions and NT region when $k=0.5, \lambda>0$ and

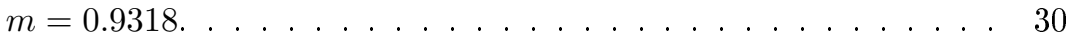

1.13 Transaction regions and NT region when $u(c)=1-e^{-\gamma c}, k>$ $0, \lambda>0$ and $m=0.9342 \ldots \ldots \ldots \ldots \ldots \ldots$

1.14 Transaction regions and NT region when $u(c)=\ln c, k>0, \lambda>$ 0 and $m=0.9342 \ldots \ldots \ldots \ldots \ldots \ldots$

2.1 Boundaries of transaction regions and NT region when $k=0 . \quad$. 46

2.2 Transaction regions when $k=0 . \ldots \ldots \ldots \ldots \ldots$

2.3 Boundaries of transaction regions and NT region when $k>0 . \ldots 47$

2.4 Transaction regions when $k>0 . \ldots \ldots \ldots \ldots$

2.5 Branch and Bound graph $\ldots \ldots \ldots \ldots \ldots$ 


\section{List of Tables}

2.1 TRT1M .......................... 48

2.2 Variance and Covariance matrix _ . . . . . . . . . . . 49

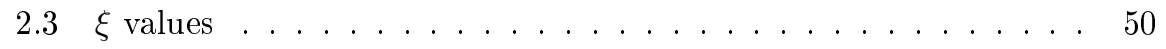

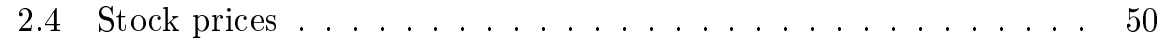




\section{Dedication}

To

my parents Mao Zhang and Yingjie Zheng

my husband Xiaofeng Wang

and

my daughter Jennifer Wang 


\section{Chapter 1}

\section{One Risky Asset Problem}

\section{$1.1 \quad$ Introduction}

We study the optimal portfolio and consumption with both the proportional and fixed transaction costs in diffusion markets. In this chapter, we restrict our attention to the infinite horizon problem with one non-risky asset (money) and one risky asset (stock). The problem is well-known. We maximize the time integral of a discounted utility function. Using the dynamic programing principle, we derive the Hamilton-Jacobi-Bellman (HJB) equation for the value function. In this chapter as a utility function we use a power function $u(c)=$ $\frac{c^{\gamma}(t)}{\gamma}(0<\gamma<1)$, where $c(t)$ is the consumption rate. We have found an explicit solution to the HJB equation. Using the explicit solution we are able to find the transaction and no-transaction regions, the transaction boundaries, and the optimal portfolio curves. Explicit solutions can be easily found for other typical utility functions such as exponential and $\log$ functions. The finite horizon problems and the case with multiple risky assets should be also treated. They will be discussed in the future publications.

Our first contribution in this chapter is the discovery of the explicit solutions to the corresponding HJB equations. To handle the transaction costs the intervention operator is introduced in $[6,14,20]$. By obtaining the explicit solutions, the intervention operator is reduced to a constrained optimization of the value functions. We accurately show the transaction regions, no-transaction region, and the transaction boundaries separating them both analytically and numerically for several different cases. Provides the existance of the explicit solutions, Verification Theorem and the Theorem of the viscosity solutions are proved. For the Verification Theorem, we show the function $\Phi$ which is defined in (1.29) satisfies (1.100), so it is the value function we seek. For the Theorem of the viscosity solutions, we prove the value function $\Phi$ is a viscosity solution for the quasi-variational inequality problem (1.30).

Our second contribution is the finding of the optimal portfolio curves and the power series solutions of the transaction boundaries curves. It is well-known that 
the optimal allocation of wealth forms a Merton line in the Merton's formulation. However, if the transaction costs are assessed, we observe that the Merton line is no longer a straight line but a curve, and moreover there are two such curves if the proportional transaction costs are considered. We also observe that notransaction region is no longer a wedge shaped region and it is a region bounded by two curves.

Our third contribution is the investors preference to the safe and risky assets. Introducing the power utility function, we let the value function be the sum of a power of the money and a power of the risky asset, which represent the investors preference to safe and risky assets. They also affect the convexity and concavity of the optimal portfolio and transaction curves. If the power of the money is greater than the power of the risky asset, the graphs of the optimal portfolio curves are concave on the $x y$-plane. This case is appropriate for conservative investors. On the other hand, if the power of the money is less than the power of the risky asset, the graphs of the optimal portfolio curves are convex on the $x y$-plane. This case is appropriate for risk-taking or entrepreneur investors.

Merton [17] is one of the earliest papers that study the optimal portfolio selection and consumption for individuals. Merton [18] extends his results from [17] for more general utility functions, price behavior assumptions, and for income generated also from noncapital gains sources. Magill and Constantinides [16] showed the importance of transaction costs, yet the wealth is treated as an independent variable. The idea of cumulative purchase and sale of the stock is introduced in Davis and Norman [8] and the money and the risky assets are distinguished. Shreve and Soner [23] considered the same problem and have shown that their value function is a viscosity solution. Their value function is basically the right hand side of (1.6). Shreve, Soner and Xu [24] studied the problem for bonds. To treat a fixed costs the quasi-variational inequalities and the associated impulse control are introduced in Eastham and Hastings [9] and refined in Korn [14]. Chancelier, Øksendal and Sulem [6] and Øksendal and Sulem [20] formulated the transaction costs problem as a combined stochastic control/impulse control problem, which in turn leads to a nonlinear quasi-variational HamiltonJacobi-Bellman inequality (QVHJBI). It is proved that the value function of the form (1.6) is the unique viscosity solution of the QVHJBI, and numerical results are presented. The viscosity solutions and their applications including the HJB equations are discussed in Fleming and Soner [10]. The optimal portfolio selection and consumption in jump diffusion markets is considered in Framstad, Øksendal and Sulem [11] and Øksendal and Sulem [21]. In Aït-Sahalia, Diaz and Hurd [1], the consumption-portfolio selection problem of an investor facing both Brownian and jump risks is analyzed, and the optimal portfolio in closed form is determined by orthogonal decompositions. A verification theorem for the quasivariational problem is proved in Brekke and Øksendal [5]. The proofs of the uniqueness for the viscosity solutions are provided in Akian, Menaldi and Sulem [2] and Akian, Sulem, and Taksar [3]. A comprehensive summary of the subject is discussed in Korn [13].

The outline of this chapter is as follows. In Section 1.2 we derive the HJB equation for the no-transaction region and define the intervention operator for 
the transaction regions. Thereby, we formulate a quasi-variational inequality problem consisting of a stochastic and an impulse control problems. Explicit solutions to the HJB equation is obtained in Section 1.3. In Section 1.4, we study analytically the optimal portfolios and the boundaries between the transaction regions and no-transaction region. We prove the verification theorem for the quasi-variational problem in Section 1.5. This verifies that the explicit solution we obtained is a value function to the optimal portfolio and consumption problem. In Section 1.6, we show that the explicit solution for the HJB equation is also a viscosity solution to the quasi-variational problem. In Section 1.7, we provide the numerical results and compare them with the analytical results obtained in Section 1.4. Explicit solutions by using exponential and natural logarithm utility functions are given in Section 1.8.

\subsection{Formulation of the problem}

We formulate a stochastic and impulse control problem for an optimal portfolio and consumption. Let $(\Omega, F, P)$ be a probability space with $F(t)(t \geq 0)$ a given filtration. We define $X(t)(t \geq 0)$ as the amount of money invested in the money market (bank) and $Y(t)(t \geq 0)$ as the stochastic process of the amount of money invested in stock. We assume that the processes $X(t)$ and $Y(t)$ satisfy

$$
\begin{gathered}
d X(t)=(r X(t)-c(t)) d t, \quad \tau_{i} \leq t \leq \tau_{i+1}, \\
d Y(t)=\alpha Y(t) d t+\sigma Y(t) d W(t), \quad \tau_{i} \leq t \leq \tau_{i+1}, \\
X\left(\tau_{i+1}\right)=X\left(\tau_{i+1}^{-}\right)-k-\xi_{i+1}-\lambda\left|\xi_{i+1}\right|, \\
Y\left(\tau_{i+1}\right)=Y\left(\tau_{i+1}^{-}\right)+\xi_{i+1},
\end{gathered}
$$

where $W(t)$ is one dimensional $F_{t}$-Brownian motion. A constant $r>0$ is the interest rate of the bank account and $c(t) \geq 0$ is the consumption rate from the bank account. Constants $\alpha>0$ and $\sigma>0$ are the mean rate of return and standard volatility of the stock respectively. We assume that $\alpha>r$. Here, $\left\{\tau_{1}, \tau_{2}, \tau_{3}, \ldots \ldots\right\}$ satisfying $0 \leq \tau_{1}<\tau_{2}<\ldots$ are $F_{t}$-stopping times at which the investor decides to change his portfolio, and $\left\{\xi_{1}, \xi_{2}, \xi_{3}, \ldots \ldots\right\}$ are sizes of the transactions at these times. In each transaction a fixed transaction cost $k \geq 0$ and a proportional transaction cost $\lambda\left|\xi_{i}\right|$ are assessed, where $\lambda(0 \leq \lambda<1)$ is a rate for the proportional transaction cost. The control of the investor is a combination of a regular stochastic control $c(t)$ and an impulse control $\phi=\left(\tau_{1}, \tau_{2}, \ldots ; \xi_{1}, \xi_{2}, \ldots\right)$. We consider the case where the shorts are not allowed. Therefore, we define a solvency region by

$$
S_{+}:=[0, \infty) \times[0, \infty)
$$

and study the problem in $S_{+}$. 
We consider the problem with the utility function $u(c)=\frac{c^{\gamma}(t)}{\gamma}$ where $\gamma$ is a constant satisfying $0<\gamma<1$. The objective of the investor is to control $(c(t), \phi)$ so as to maximize the time integral of the discounted utility function for his life time consumption or to find the value function

$$
\widetilde{\Phi}(s, x, y)=\sup _{c, \phi} E\left[\int_{0}^{\infty} e^{-\delta(s+t)} \frac{c^{\gamma}(t)}{\gamma} d t\right],
$$

where $\delta>0$ is a constant discount rate and we assume

$$
\alpha>\delta>r .
$$

In other words, the discount rate (or inflation rate) is larger than the interest rate and smaller than the growth rate of the risky asset. Note that $1-\gamma$ is called the relative risk aversion coefficient. In the infinite horizon problem we can simplify the problem in the following manner

$$
\widetilde{\Phi}(s, x, y)=e^{-\delta s} \sup _{c, \phi} E\left[\int_{0}^{\infty} e^{-\delta t} \frac{c^{\gamma}(t)}{\gamma} d t\right]=e^{-\delta s} \widetilde{\Phi}(0, x, y) \triangleq e^{-\delta s} \Phi(x, y)
$$

so that we seek a time independent value function $\Phi(x, y)$.

We derive the equations governing the value function in the rest of this section and in the next section define the explicit form of the value function. We need to distinguish the case where there are no transactions and the case where the transactions are taking place. First we discuss the no-transaction case. Denoting by $\tilde{v}(s, x, y)=e^{-\delta s} v(x, y)$ the value function for the no-transaction case and using the dynamical principle, we see that $v$ satisfies the HamiltonJacobi-Bellman (HJB) equation given by

$$
\max _{c}\left[-\delta v+(r x-c) \frac{\partial v}{\partial x}+\alpha y \frac{\partial v}{\partial y}+\frac{1}{2} \sigma^{2} y^{2} \frac{\partial^{2} v}{\partial y^{2}}+\frac{c^{\gamma}}{\gamma}\right]=0 .
$$

We define

$$
L^{c} v(x, y)=-\delta v+(r x-c) \frac{\partial v}{\partial x}+\alpha y \frac{\partial v}{\partial y}+\frac{1}{2} \sigma^{2} y^{2} \frac{\partial^{2} v}{\partial y^{2}} .
$$

Also, we define

$$
\begin{gathered}
L v(x, y)=\sup _{c \geq 0}\left\{L^{c} v(x, y)+\frac{c^{\gamma}}{\gamma}\right\}, \quad(x, y) \in S_{+} . \\
L_{0} v(0, y)=-\delta v+\alpha y \frac{\partial v}{\partial y}+\frac{1}{2} \sigma^{2} y^{2} \frac{\partial^{2} v}{\partial y^{2}} \\
L_{1} v(x, 0)=\sup _{c \geq 0}\left\{-\delta v+(r x-c) \frac{\partial v}{\partial x}+\frac{c^{\gamma}}{\gamma}\right\} .
\end{gathered}
$$


To handle the transactions we introduce an intervention operator $M$ defined by

$$
M v(x, y)=\sup _{\xi}\left\{v\left(x^{\prime}, y^{\prime}\right) \mid \xi \in R \backslash\{0\},\left(x^{\prime}, y^{\prime}\right) \in S_{+}\right\}
$$

where

$$
\begin{gathered}
x^{\prime}=x^{\prime}(\xi)=x-k-\xi-\lambda|\xi|, \\
y^{\prime}=y^{\prime}(\xi)=y+\xi .
\end{gathered}
$$

Here, $k$ and $\lambda$ are a fixed and a proportional transaction costs of trading, respectively and (1.15) gives a line of trading. This is a constrained optimization of the value function. We compare the current value $v$ and the optimal value $M v$ after the trading. The region where $M v \geq v$ is called the transaction region and the region where $M v<v$ is called the no-transaction region and denoted by $N T$, i.e.,

$$
N T=\left\{(x, y) \in S_{+} ; v(x, y)>M v(x, y)\right\} .
$$

We also call the set of $(x, y) \in S_{+}$at which $M v(x, y)=v(x, y)$ the transaction boundaries.

\subsection{Solutions to HJB equation}

We consider the equation (1.9) first. Concerning the solutions of (1.9), we have

Theorem 1.1. A solution to (1.9) is given by

$$
v(x, y)=a x^{\gamma}+\frac{y^{m}}{m},
$$

where

$$
a=\frac{1}{\gamma}\left[\frac{1}{1-\gamma}(\delta-r \gamma)\right]^{\gamma-1}
$$

and

$$
m=\frac{\left(\frac{1}{2} \sigma^{2}-\alpha\right) \pm \sqrt{\left(\frac{1}{2} \sigma^{2}-\alpha\right)^{2}+2 \sigma^{2} \delta}}{\sigma^{2}} .
$$

Proof. Differentiating (1.9) with respect to $c$, we have $c^{*}=\left(\frac{\partial v}{\partial x}\right)^{\frac{1}{\gamma-1}}$, and substituting $c^{*}$ into (1.9), we obtain

$$
-\delta v+r x \frac{\partial v}{\partial x}+\alpha y \frac{\partial v}{\partial y}+\frac{1}{2} \sigma^{2} y^{2} \frac{\partial^{2} v}{\partial y^{2}}+\frac{1-\gamma}{\gamma}\left(\frac{\partial v}{\partial x}\right)^{\frac{\gamma}{\gamma-1}}=0 .
$$

Substituting the ansatz (1.18) in (1.21) and collecting the like terms, we obtain two equations

$$
\left[-\delta a+r a \gamma+\frac{1-\gamma}{\gamma} a^{\frac{\gamma}{\gamma-1}} \gamma^{\frac{\gamma}{\gamma-1}}\right] x^{\gamma}=0,
$$

and

$$
\left[\frac{1}{2} \sigma^{2} m^{2}+\left(\alpha-\frac{1}{2} \sigma^{2}\right) m-\delta\right] \frac{y^{m}}{m}=0 .
$$


Since they hold for arbitrary nonnegative $x$ and $y$, the coefficients must be zero. Therefore,

$$
-\delta a+r a \gamma+\frac{1-\gamma}{\gamma} a^{\frac{\gamma}{\gamma-1}} \gamma^{\frac{\gamma}{\gamma-1}}=0,
$$

and

$$
\frac{1}{2} \sigma^{2} m^{2}+\left(\alpha-\frac{1}{2} \sigma^{2}\right) m-\delta=0 .
$$

It is easy to obtain (1.19) from (1.24). For $m$, solving (1.25), we have

$$
m=\frac{\left(\frac{1}{2} \sigma^{2}-\alpha\right) \pm \sqrt{\left(\frac{1}{2} \sigma^{2}-\alpha\right)^{2}+2 \sigma^{2} \delta}}{\sigma^{2}} .
$$

Set $g(m)=\frac{1}{2} \sigma^{2} m^{2}+\left(\alpha-\frac{1}{2} \sigma^{2}\right) m-\delta$, and notice that

$$
g(0)=-\delta<0, \quad g(1)=\alpha-\delta>0 .
$$

The intermediate value theorem implies that the positive $m$ satisfies $0<m<$ 1.

Remark 1.2. Note that $v(x, y)=a x^{\gamma}+b y^{m}$, where $a$ is given by (1.19) and $b$ is a constant satisfying $b m>0$, is also a solution. The condition $b m>0$ guarantees that the value function is concave. Therefore, following the same practice as for the utility function, we choose $b=\frac{1}{m}$. In what follows, we denote

$$
m_{ \pm}=\frac{\left(\frac{1}{2} \sigma^{2}-\alpha\right) \pm \sqrt{\left(\frac{1}{2} \sigma^{2}-\alpha\right)^{2}+2 \sigma^{2} \delta}}{\sigma^{2}} .
$$

Now let us look at the second part of equation (1.30). For a given $\zeta=$ $(x, y) \in S_{+}$, in order to compute

$$
M v=\sup v\left(x^{\prime}, y^{\prime}\right)
$$

with $x^{\prime}=x-k-\xi-\lambda|\xi|, y^{\prime}=y+\xi$, we need to find the value of $\xi$ that will maximize the value function

$$
v\left(x^{\prime}, y^{\prime}\right)=a x^{\prime \gamma}+\frac{y^{\prime m}}{m}= \begin{cases}a(x-k-\xi-\lambda \xi)^{\gamma}+\frac{(y+\xi)^{m}}{m} & (\xi>0) \\ a(x-k-\xi+\lambda \xi)^{\gamma}+\frac{(y+\xi)^{m}}{m} . & (\xi<0) .\end{cases}
$$

Since we know an explicit solution in the no transaction region, the problem is reduced to finding

$$
\max [v(x, y), M v(x, y)]
$$

for each $(x, y) \in S_{+}$.

Combining the no-transaction and transaction cases, we define

$$
\Phi(x, y)=\max [v(x, y), M v(x, y)]
$$


as the value function. Therefore, we obtain a quasi-variational inequality problem

$$
\max _{c, \phi}\left[L^{c} v+\frac{c^{\gamma}}{\gamma}, M v-\Phi(x, y)\right]=0
$$

with the understanding that if both $L^{c} v+\frac{1}{\gamma} c^{\gamma}$ and $M v-\Phi(x, y)$ are equal to zero, we make trades and move to a new allocation. With this formulation we can handle both fixed and proportional transaction costs.

\subsection{Analytical study of the boundaries between transaction regions and NT-region}

We study the transaction regions, no-transaction region (NT-region), and the transaction boundaries separating them. First we explain various notations in Figure 1.1. The line $L_{1}$ is the line along which the risky asset is sold. The curve $C_{1}$ is the set of $\left(x_{0}, y_{0}\right)$ at which the value function attains its maximum along the line $L_{1}$ for each $\left(x_{1}, y_{1}\right)$ given on the left of $C_{1}$. The curve $B_{1}$ is the set of $(\hat{x}, \hat{y})$ on the boundary between the sell region and the NT-region. Note that $\left(x_{0}, y_{0}\right)$ is the intersection of curves $C_{1}$ and a contour curve of the value function denoted as $V F C$, while $(\hat{x}, \hat{y})$ is the intersection of the curves $B_{1}$ and $V F C$. We call $B_{1}$ the transaction boundary and call $C_{1}$ the optimal portfolio curve. Similarly, we define $L_{2}$ to be the line along which the risky asset is bought, and $B_{2}$ and $C_{2}$ are the corresponding transaction boundary and optimal portfolio curve, respectively. In what follows, we obtain the relations among $(\hat{x}, \hat{y})$ and $\left(x_{0}, y_{0}\right)$ in selling the risky asset.

Since the value function is a constant along each $V F C$ and both $\left(x_{0}, y_{0}\right)$ and $(\hat{x}, \hat{y})$ are on the same $V F C$, we obtain

$$
a \hat{x}^{\gamma}+\frac{\hat{y}^{m}}{m}=a x_{0}^{\gamma}+\frac{y_{0}^{m}}{m} .
$$

Also, the fact that $(\hat{x}-k, \hat{y})$ is on the line $L_{1}$ implies

$$
\hat{x}-k-x_{0}+(1-\lambda)\left(\hat{y}-y_{0}\right)=0 .
$$

The tangent line to the curve $V F C$ at $\left(x_{0}, y_{0}\right)$ agrees with the line of trading $L_{1}$. Therefore, equating the slope of the tangent line to $V F C$ at $\left(x_{0}, y_{0}\right)$ and the slope of the trading line, we obtain the optimal portfolio curve $C_{1}$

$$
-\frac{1}{1-\lambda}=-\frac{a \gamma x_{0}^{\gamma-1}}{y_{0}^{m-1}} \quad \text { or } \quad y_{0}^{1-m}=\frac{1}{a \gamma(1-\lambda)} x_{0}^{1-\gamma} .
$$

Now we have four variables $\hat{x}, \hat{y}, x_{0}, y_{0}$, and three equations (1.31), (1.32), and (1.33). We express $\hat{x}$ and $\hat{y}$ in terms of $x_{0}, y_{0}$ and $k$.

As a passing note, we see that the optimal portfolio curve $C_{2}$ is given by

$$
y_{0}^{1-m}=\frac{1}{a \gamma(1+\lambda)} x_{0}^{1-\gamma} .
$$




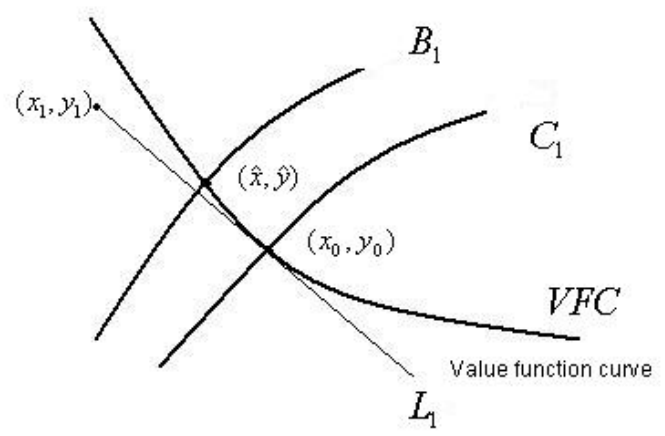

Figure 1.1: Graphs for several curves.

Theorem 1.3. There is a saddle node type bifurcation of the solution $(\widehat{x}, \widehat{y})$ from $\left(x_{0}, y_{0}\right)$ as we increase $k$ from zero. One of the bifurcating solutions $(\widehat{x}, \widehat{y})$ forms the transaction boundary and is given by the power series in $k^{\frac{1}{2}}$, i.e.,

$$
\widehat{x}=x_{0}+\sum_{n=1}^{\infty} a_{n} k^{\frac{n}{2}}, \quad \widehat{y}=y_{0}+\sum_{n=1}^{\infty} b_{n} k^{\frac{n}{2}},
$$

where the first few terms of $a_{n}$ and $b_{n}$ are given in (1.47) and (1.50).

Proof. We find the power series solution to

$$
v(x, y)=a \hat{x}^{\gamma}+\frac{1}{m} \hat{y}^{m}=a x_{0}^{\gamma}+\frac{1}{m} y_{0}^{m}
$$

and

$$
\left(\hat{x}-k-x_{0}\right)+(1-\lambda)\left(\hat{y}-y_{0}\right)=0
$$

when

$$
(1-\lambda) a \gamma x_{0}^{\gamma-1}=y_{0}^{m-1} .
$$

We assume that the power series is given by

$$
x=x_{0}+\sum_{n=1}^{\infty} a_{n} k^{n p}, \quad y=y_{0}+\sum_{n=1}^{\infty} b_{n} k^{n p} .
$$

We expand $v$ about $\left(x_{0}, y_{0}\right)$

$$
\begin{aligned}
& v_{x}\left(x-x_{0}\right)+\frac{1}{2} v_{x x}\left(x-x_{0}\right)^{2}+\frac{1}{3 !} v_{x x x}\left(x-x_{0}\right)^{3}+\cdots \\
& +v_{y}\left(y-y_{0}\right)+\frac{1}{2} v_{y y}\left(y-y_{0}\right)^{2}+\frac{1}{3 !} v_{y y y}\left(y-y_{0}\right)^{3}+\cdots=0,
\end{aligned}
$$


where the partial derivatives of $v$ are evaluated at $\left(x_{0}, y_{0}\right)$. Then, from (1.39) and (1.40)

$$
\begin{aligned}
& v_{x}\left(\sum_{n=1}^{\infty} a_{n} k^{n p}\right)+\frac{1}{2} v_{x x}\left(\sum_{n=1}^{\infty} a_{n} k^{n p}\right)^{2}+\frac{1}{3 !} v_{x x x}\left(\sum_{n=1}^{\infty} a_{n} k^{n p}\right)^{3}+\cdots \\
& +v_{y}\left(\sum_{n=1}^{\infty} b_{n} k^{n p}\right)+\frac{1}{2} v_{y y}\left(\sum_{n=1}^{\infty} b_{n} k^{n p}\right)^{2}+\frac{1}{3 !} v_{y y y}\left(\sum_{n=1}^{\infty} b_{n} k^{n p}\right)^{3}+\cdots \\
= & 0 .
\end{aligned}
$$

Also, from (1.37) and (1.39)

$$
\left(\sum_{n=1}^{\infty} a_{n} k^{n p}\right)+(1-\lambda)\left(\sum_{n=1}^{\infty} b_{n} k^{n p}\right)=k .
$$

For $n=1$ if $p=1$, collecting the $O(k)$ terms in (1.41) and (1.42), we have

$$
A\left[\begin{array}{l}
a_{1} \\
b_{1}
\end{array}\right]=\left[\begin{array}{cc}
v_{x} & v_{y} \\
1 & 1-\lambda
\end{array}\right]\left[\begin{array}{l}
a_{1} \\
b_{1}
\end{array}\right]=\left[\begin{array}{l}
0 \\
1
\end{array}\right] .
$$

Because of (1.38) the matrix $A$ is singular. Therefore, to have a solution, by the Fredholm alternative the right hand side must be orthogonal to the left nullspace of $A$. We take $\left[-1 v_{x}\right]$ to be the left nullspace. Then, multiplying $\left[\begin{array}{ll}-1 & v_{x}\end{array}\right]$ from the left, we see that $v_{x}=a \gamma x_{0}^{\gamma-1}=0$. Therefore, $p$ can not be one and the right hand side of (1.43) is zero. So, $\left(a_{1}, b_{1}\right)$ are undetermined from $n=1$.

For $n=2$ if we choose $p=\frac{1}{2}$, from (1.41) and (1.42) we have

$$
A\left[\begin{array}{l}
a_{2} \\
b_{2}
\end{array}\right]=\left[\begin{array}{c}
-\frac{1}{2} v_{x x} a_{1}^{2}-\frac{1}{2} v_{y y} b_{1}^{2} \\
1
\end{array}\right] .
$$

Applying the same argument as $n=1$, we have

$$
\frac{1}{2} v_{x x}(1-\lambda)^{2} b_{1}^{2}+\frac{1}{2} v_{y y} b_{1}^{2}+v_{x}=0
$$

and therefore,

$$
b_{1}^{2}=-\frac{2 v_{x}}{v_{x x}(1-\lambda)^{2}+v_{y y}}=\frac{2}{(1-\gamma) x_{0}^{-1}(1-\lambda)^{2}+(1-m)(1-\lambda) y_{0}^{-1}} .
$$

Denote the denominator as $D_{1}$. Then,

$$
b_{1}= \pm \sqrt{\frac{2}{D_{1}}}, \quad a_{1}=\mp(1-\lambda) \sqrt{\frac{2}{D_{1}}} .
$$

For $a_{1}$ we choose the negative and positive sign for selling and buying, respectively, while for $b_{1}$ we choose the positive and negative sign for selling and buying, respectively. Since $\left(a_{1}, b_{1}\right)$ are determinable for $p=\frac{1}{2}$, we choose $p=\frac{1}{2}$ 
and continue the process where at the $n$th step we determine $\left(a_{n-1}, b_{n-1}\right)$ by the Fredholm alternative.

For $n=3$, collecting the $O\left(k^{\frac{3}{2}}\right)$ terms in (1.41) and (1.42), we have

$$
A\left[\begin{array}{l}
a_{3} \\
b_{3}
\end{array}\right]=-\left[\begin{array}{c}
\frac{1}{2} v_{x x}\left(2 a_{1} a_{2}\right)+\frac{1}{3 !} v_{x x x} a_{1}^{3}+\frac{1}{2} v_{y y}\left(2 b_{1} b_{2}\right)+\frac{1}{3 !} v_{y y y} b_{1}^{3} \\
0
\end{array}\right] .
$$

Using the Fredholm alternative and $a_{2}+(1-\lambda) b_{2}=1$, we have

$$
\left[\begin{array}{cc}
v_{x x} a_{1} & v_{y y} b_{1} \\
1 & (1-\lambda)
\end{array}\right]\left[\begin{array}{c}
a_{2} \\
b_{2}
\end{array}\right]=\left[\begin{array}{c}
-\frac{1}{3 !}\left(v_{x x x} a_{1}^{3}+v_{y y y} b_{1}^{3}\right) \\
1
\end{array}\right] .
$$

Denote the determinant of the matrix on the left hand side by

$$
D_{2}=(1-\lambda)^{2} a \gamma(1-\gamma) x_{0}^{\gamma-2}+(1-m) y_{0}^{m-2}
$$

- Then,

$$
\left[\begin{array}{l}
a_{2} \\
b_{2}
\end{array}\right]=\frac{1}{D_{2}}\left[\begin{array}{c}
\frac{1}{3 !}(1-\lambda)\left((1-\lambda) v_{x x x} a_{1}^{2}-v_{y y y} b_{1}^{2}\right)-v_{y y} \\
-\frac{1}{3 !}(1-\lambda) v_{x x x} a_{1}^{2}+\frac{1}{3 !} v_{y y y} b_{1}^{2}-(1-\lambda) v_{x x}
\end{array}\right]
$$

For $n=n$, collecting the $O\left(k^{\frac{n}{2}}\right)$ temrs in (1.41) and (1.42), we have

$$
\begin{aligned}
& A\left[\begin{array}{c}
a_{n} \\
b_{n}
\end{array}\right] \\
= & -\left[\begin{array}{c}
f\left(a_{1}, a_{2}, \ldots, a_{n-1}, b_{1}, b_{2}, \ldots, b_{n-1}\right) \\
0
\end{array}\right],
\end{aligned}
$$

where

$$
\begin{aligned}
& f\left(a_{1}, a_{2}, \ldots, a_{n-1}, b_{1}, b_{2}, \ldots, b_{n-1}\right) \\
= & v_{x x} a_{1} a_{n-1}+v_{x x} a_{2} a_{n-2}+\frac{1}{2} v_{x x x} a_{1}^{2} a_{n-2} \\
+ & v_{y y} b_{1} b_{n-1}+\frac{1}{2} v_{y y y} b_{1}^{2} b_{n-2}+\cdots
\end{aligned}
$$

Using $a_{n-1}+(1-\lambda) b_{n-1}=0$ and the Fredholm alternative, we obtain

$$
B\left[\begin{array}{l}
a_{n-1} \\
b_{n-1}
\end{array}\right]=-\left[\begin{array}{c}
v_{x x} a_{2} a_{n-2}+\frac{1}{2}\left(v_{x x x} a_{1}^{2} a_{n-2}+v_{y y y} b_{1}^{2} b_{n-2}\right)+\cdots \\
0
\end{array}\right] .
$$

Since the determinant of $B$ is nonzero, the procedure can be continued.

Theorem 1.4. The transaction boundaries are concave downward if $m<\gamma$, and are concave upward if $m>\gamma$. 
Proof. We prove the case where $m<\gamma$. The case where $m>\gamma$ is proved in a similar manner. Substituting (1.33) to (1.31) and (1.32), we obtain

$$
R_{1}\left(\hat{x}, x_{0}, \hat{y}\right)=a \hat{x}^{\gamma}+\frac{\hat{y}^{m}}{m}-a x_{0}^{\gamma}-\frac{[(1-\lambda) a \gamma]^{\frac{m}{m-1}} \cdot x_{0}^{\frac{m(\gamma-1)}{m-1}}}{m},
$$

and

$$
R_{2}\left(\hat{x}, x_{0}, \hat{y}\right)=\left(\hat{x}-k-x_{0}\right)+(1-\lambda)\left\{\hat{y}-[(1-\lambda) a \gamma]^{\frac{1}{m-1}} \cdot x_{0}^{\frac{\gamma-1}{m-1}}\right\} .
$$

We regard $x_{0}$ and $\hat{y}$ are functions of $\hat{x}$ and differentiate (1.54) and (1.55) with respect to $\hat{x}$. Then

$$
a \gamma \hat{x}^{\gamma-1}+\hat{y}^{m-1} \cdot \frac{d \hat{y}}{d \hat{x}}-A_{1} \cdot \frac{d x_{0}}{d \hat{x}}=0
$$

and

$$
1+(1-\lambda) \frac{d \hat{y}}{d \hat{x}}-A_{2} \cdot \frac{d x_{0}}{d \hat{x}}=0
$$

where

$$
\begin{array}{r}
A_{1}=a \gamma x_{0}^{\gamma-1}+\frac{[(1-\lambda) a \gamma]^{\frac{m}{m-1}}}{m} \cdot \frac{m(\gamma-1)}{m-1} \cdot x_{0}^{\left[\frac{m(\gamma-1)}{m-1}-1\right]}, \\
A_{2}=1+(1-\lambda)[(1-\lambda) a \gamma]^{\frac{1}{m-1}} \cdot \frac{\gamma-1}{m-1} \cdot x_{0}^{\left[\frac{\gamma-1}{m-1}-1\right]} .
\end{array}
$$

Eliminating $\frac{d x_{0}}{d \hat{x}}$ from (1.56) and (1.57), and using the relation $A_{1}=a \gamma x_{0}^{\gamma-1} \cdot A_{2}$, we obtain

$$
\frac{d \hat{y}}{d \hat{x}}=\frac{a \gamma x_{0}^{\gamma-1}-a \gamma \hat{x}^{\gamma-1}}{\hat{y}^{m-1}-y_{0}^{m-1}} .
$$

This yields

$$
\frac{d^{2} \hat{y}}{d \hat{x}^{2}}=\frac{1}{\left(\hat{y}^{m-1}-y_{0}^{m-1}\right)^{2}}\left\{T_{1}+T_{2}+T_{3}+T_{4}\right\}
$$

where

$$
\begin{gathered}
T_{1}=a \gamma(\gamma-1) x_{0}^{\gamma-2} \frac{1}{A_{2}}\left[\hat{y}^{m-1}-(1-\lambda) a \gamma \hat{x}^{\gamma-1}\right], \\
T_{2}=-a \gamma(\gamma-1) \hat{x}^{\gamma-2} \cdot\left(\hat{y}^{m-1}-y_{0}^{m-1}\right), \\
T_{3}=-(m-1) \frac{\hat{y}^{m-2}}{\hat{y}^{m-1}-y_{0}^{m-1}}\left(a \gamma x_{0}^{\gamma-1}-a \gamma \hat{x}^{\gamma-1}\right)^{2},
\end{gathered}
$$

and

$$
T_{4}=a \gamma(\gamma-1) \frac{x_{0}^{\gamma-1}-\hat{x}^{\gamma-1}}{\hat{y}^{m-1}-y_{0}^{m-1}}[(1-\lambda) a \gamma] x_{0}^{\gamma-2} \cdot \frac{1}{A_{2}}\left[\hat{y}^{m-1}-(1-\lambda) a \gamma \hat{x}^{\gamma-1}\right] .
$$


Since $(\hat{x}, \hat{y})$ is on $B_{1}$ and $\left(x_{0}, y_{0}\right)$ is on $C_{1}, \hat{x}<x_{0}, \hat{y}>y_{0}$. Therefore, since $0<\gamma<1$ and $0<m<1$, we see that

$$
\hat{x}^{\gamma-2}>x_{0}^{\gamma-2}
$$

and

$$
\hat{y}^{m-1}<y_{0}^{m-1} .
$$

Using $\frac{1}{A_{2}}<1$, we estimate $T_{1}+T_{4}$ as follows.

$$
\begin{aligned}
T_{1}+T_{4} & =a \gamma(\gamma-1) x_{0}^{\gamma-2} \frac{1}{A_{2}} \cdot \frac{\left[\hat{y}^{m-1}-(1-\lambda) a \gamma \hat{x}^{\gamma-1}\right]^{2}}{\hat{y}^{m-1}-y_{0}^{m-1}} \\
& <a \gamma(\gamma-1) x_{0}^{\gamma-2} \cdot \frac{\left[\hat{y}^{m-1}-(1-\lambda) a \gamma \hat{x}^{\gamma-1}\right]^{2}}{\hat{y}^{m-1}-y_{0}^{m-1}} \\
& <a \gamma(\gamma-1) \hat{x}^{\gamma-2} \cdot \frac{\left[\hat{y}^{m-1}-(1-\lambda) a \gamma x_{0}^{\gamma-1}\right]^{2}}{\hat{y}^{m-1}-y_{0}^{m-1}} \\
& =\left|T_{2}\right| .
\end{aligned}
$$

Then, since $T_{2}<0, T_{3}<0$, and $0<T_{1}+T_{4}<\left|T_{2}\right|, T_{1}+T_{2}+T_{3}+T_{4}<0$. Therefore,

$$
\frac{d^{2} \hat{y}}{d \hat{x}^{2}}<0
$$

In what follows, we denote the transaction boudaries $B_{1}$ and $B_{2}$ by $y=h_{s}(x)$ and $y=h_{b}(x)$, respectively. After $B_{2}$ reaches the $x$-axis, $h_{b}(x)$ is continued as zero till the origin.

\subsection{Verification Theorem}

To show the verification theorem it is necessary to study the processes $X, Y$, and the discounted utility between the two consecutive transaction times $\tau_{i}^{+}$ and $\tau_{i+1}^{-}$. We start from $X$.

Lemma 1.5. The amount of money and the discounted utility of money decrease between the two consecutive transaction times.

Proof. Since

$$
c^{*}=(a \gamma)^{\frac{1}{\gamma-1}} x=\frac{(\delta-r \gamma)}{(1-\gamma)} x,
$$

for $\tau_{i}^{+}<t<\tau_{i+1}^{-}$,

$$
d X(t)=(r X(t)-c(t)) d t=\left(r-\frac{(\delta-r \gamma)}{(1-\gamma)}\right) X(t) d t=\frac{(r-\delta)}{(1-\gamma)} X(t) d t
$$


By assumption (1.7), (1.70) implies that the amount of money decreases for $\tau_{i}^{+}<t<\tau_{i+1}^{-}$. For the discounted utility of money we have

$$
d\left(e^{-\delta t} a \cdot X^{\gamma}(t)\right)=-\frac{(\delta-r \gamma)}{(1-\gamma)} e^{-\delta t} a \cdot X^{\gamma}(t) d t, \tau_{i}^{+}<t<\tau_{i+1}^{-} .
$$

Integrating in $t$ from $\tau_{i}^{+}$to $\tau_{i+1}^{-}$, we obtain

$$
\begin{aligned}
& e^{-\delta \tau_{i+1}^{-}} a \cdot X^{\gamma}\left(\tau_{i+1}^{-}\right)-e^{-\delta \tau_{i}^{+}} a \cdot X^{\gamma}\left(\tau_{i}^{+}\right) \\
= & e^{-\delta \tau_{i}^{+}} a \cdot X^{\gamma}\left(\tau_{i}^{+}\right)\left[e^{-\frac{(\delta-r \gamma)}{(1-\gamma)}\left(\tau_{i+1}^{-}-\tau_{i}^{+}\right)}-1\right] .
\end{aligned}
$$

Since $\delta-r \gamma>0$, the right hand side is negative.

Next consider $Y$. We have

Lemma 1.6. The discounted utility of risky asset satisfies

$$
e^{-\delta \tau_{i+1}^{-}} \frac{Y^{m}\left(\tau_{i+1}^{-}\right)}{m}-e^{-\delta \tau_{i}^{+}} \frac{Y^{m}\left(\tau_{i}^{+}\right)}{m}=e^{-\delta \tau_{i}^{+}} \frac{Y^{m}\left(\tau_{i}^{+}\right)}{m}\left[e^{M_{i}}-1\right]
$$

where

$$
M_{i}=\left\{\left[m\left(\alpha-\frac{1}{2} \sigma^{2}\right)-\delta\right]\left(\tau_{i+1}^{-}-\tau_{i}^{+}\right)+m \sigma\left[W\left(\tau_{i+1}^{-}\right)-W\left(\tau_{i}^{+}\right)\right]\right\} .
$$

Furthermore, the expectation of $e^{M_{i}}$ is one.

Proof. Since $Y$ follows the geometric Brownian motion for $\tau_{i}^{+} \leq x<\tau_{i+1}^{-}$, $Y\left(\tau_{i+1}^{-}\right)$is expressed as

$$
Y\left(\tau_{i+1}^{-}\right)=Y\left(\tau_{i}^{+}\right) e^{\left\{\left(\alpha-\frac{1}{2} \sigma^{2}\right)\left(\tau_{i+1}^{-}-\tau_{i}^{+}\right)+\sigma\left[W\left(\tau_{i+1}^{-}\right)-W\left(\tau_{i}^{+}\right)\right]\right\}}, i=0,1,2, \ldots, N .
$$

Also, the Ito-Doeblin formula implies that the discounted value function of the risky asset is given by

$$
\begin{aligned}
& e^{-\delta \tau_{i+1}^{-}} \frac{Y^{m}\left(\tau_{i+1}^{-}\right)}{m} \\
= & e^{-\delta \tau_{i}^{+}} \frac{Y^{m}\left(\tau_{i}^{+}\right)}{m} e^{\left\{\left[m\left(\alpha-\frac{1}{2} \sigma^{2}\right)-\delta\right]\left(\tau_{i+1}^{-}-\tau_{i}^{+}\right)+m \sigma\left[W\left(\tau_{i+1}^{-}\right)-W\left(\tau_{i}^{+}\right)\right]\right\}} .
\end{aligned}
$$

Therefore, we obtain (1.73).

The expectation of $e^{M_{i}}$ is given by

$$
E\left\{e^{M_{i}}\right\}=\frac{1}{\sqrt{2 \pi}} \int_{-\infty}^{\infty} e^{\left[m\left(\alpha-\frac{1}{2} \sigma^{2}\right)-\delta\right]\left(\tau_{i+1}^{-}-\tau_{i}^{+}\right)+m \sigma \sqrt{\tau_{i+1}^{-}-\tau_{i}^{+}} z_{i}-\frac{1}{2} z_{i}^{2}} d z_{i} .
$$

By (1.23) the exponent of integrand is simplified as follows

$$
\begin{aligned}
& {\left[m\left(\alpha-\frac{1}{2} \sigma^{2}\right)-\delta\right]\left(\tau_{i+1}^{-}-\tau_{i}^{+}\right)+m \sigma \sqrt{\tau_{i+1}^{-}-\tau_{i}^{+}} z_{i}-\frac{1}{2} z_{i}^{2} } \\
= & {\left[m\left(\alpha-\frac{1}{2} \sigma^{2}\right)-\delta+\frac{1}{2} m^{2} \sigma^{2}\right]\left(\tau_{i+1}^{-}-\tau_{i}^{+}\right)-\frac{1}{2}\left(z_{i}-m \sigma \sqrt{\tau_{i+1}^{-}-\tau_{i}^{+}}\right)^{2} } \\
= & -\frac{1}{2}\left(z_{i}-m \sigma \sqrt{\tau_{i+1}^{-}-\tau_{i}^{+}}\right)^{2}
\end{aligned}
$$


and consequently we have

$$
E\left\{e^{M_{i}}\right\}=\frac{1}{\sqrt{2 \pi}} \int_{-\infty}^{\infty} e^{-\frac{1}{2}\left(z_{i}-m \sigma \sqrt{\tau_{i+1}^{-}-\tau_{i}^{+}}\right)^{2}} d z_{i}=1
$$

We need to know an estimate for the probability of staying in the notransaction region or reaching the transaction boundary at $\tau_{i+1}^{-}$starting from $\left(X\left(\tau_{i}^{+}\right), Y\left(\tau_{i}^{+}\right)\right)$. From (1.75), define

$$
D\left(\tau_{i}^{+}, Y\left(\tau_{i+1}^{-}\right)\right)=\frac{\log \frac{Y\left(\tau_{i+1}^{-}\right)}{Y\left(\tau_{i}^{+}\right)}-\left(\alpha-\frac{1}{2} \sigma^{2}\right)\left(\tau_{i+1}^{-}-\tau_{i}^{+}\right)}{\sigma \sqrt{\tau_{i+1}^{-}-\tau_{i}^{+}}} .
$$

Lemma 1.7. The probability $P_{s}$ of staying in the no-transaction region at $\tau_{i+1}^{-}$ is estimated as

$$
P_{s} \leq \frac{1}{\sqrt{2 \pi}} \int_{D\left(\tau_{i}^{+}, h_{b}\left(X\left(\tau_{i+1}^{-}\right)\right)\right.}^{D\left(\tau_{i}^{+}, h_{s}\left(X\left(\tau_{i+1}^{-}\right)\right)\right.} e^{-\frac{1}{2} z_{i}^{2}} d z_{i} .
$$

Also, the probability $P_{o}$ of leaving the no-transaction region by $\tau_{i+1}^{-}$is estimated as

$$
P_{o} \leq \frac{2}{\sqrt{2 \pi}} \int_{D\left(\tau_{i}^{+}, h_{s}\left(X\left(\tau_{i+1}^{-}\right)\right)\right.}^{\infty} e^{-\frac{1}{2} z_{i}^{2}} d z_{i}+\frac{2}{\sqrt{2 \pi}} \int_{-\infty}^{D\left(\tau_{i}^{+}, h_{b}\left(X\left(\tau_{i}^{+}\right)\right)\right.} e^{-\frac{1}{2} z_{i}^{2}} d z_{i}
$$

Furthermore, $P_{o} \rightarrow 0$ as $\tau_{i+1}^{-} \rightarrow \tau_{i}^{+}$and this implies that the probability of immediate transaction after a transaction is zero.

Proof. To stay in the no-transaction region till $\tau_{i+1}^{-}, Z_{i}$ must satisfy

$$
D\left(\tau_{i}^{+}, h_{b}\left(X\left(\tau_{i+1}^{-}\right)\right)<Z_{i}<D\left(\tau_{i}^{+}, h_{s}\left(X\left(\tau_{i+1}^{-}\right)\right) .\right.\right.
$$

Since $Z_{i}$ follows the standard normal distribution, this implies (1.81). The probability of $Z_{i}$ being the outside of the no-transaction region at $\tau_{i+1}^{-}$is

$$
\begin{aligned}
& P\left\{Z_{i} \geq D\left(\tau_{i}^{+}, h_{s}\left(X\left(\tau_{i+1}^{-}\right)\right) \text {or } Z_{i} \leq D\left(\tau_{i}^{+}, h_{b}\left(X\left(\tau_{i}^{+}\right)\right)\right\}\right.\right. \\
= & \frac{1}{\sqrt{2 \pi}} \int_{D\left(\tau_{i}^{+}, h_{s}\left(X\left(\tau_{i+1}^{-}\right)\right)\right.}^{\infty} e^{-\frac{1}{2} z_{i}^{2}} d z_{i} \\
& +\frac{1}{\sqrt{2 \pi}} \int_{-\infty}^{D\left(\tau_{i}^{+}, h_{b}\left(X\left(\tau_{i}^{+}\right)\right)\right.} e^{-\frac{1}{2} z_{i}^{2}} d z_{i} .
\end{aligned}
$$

By the reflection principle, the probability of leaving the no-transaction region by $\tau_{i+1}^{-}$is less than the twice of the above probability. Therefore, we obtain (1.82).

Since $Y\left(\tau_{i}^{+}\right)<h_{s}\left(X\left(\tau_{i}^{+}\right), D\left(\tau_{i}^{+}, h_{s}\left(X\left(\tau_{i+1}^{-}\right)\right) \rightarrow \infty\right.\right.$ as $\tau_{i+1}^{-} \rightarrow \tau_{i}^{+}$. Similarly, $D\left(\tau_{i}^{+}, h_{b}\left(X\left(\tau_{i}^{+}\right)\right) \rightarrow-\infty\right.$ as $\tau_{i+1}^{-} \rightarrow \tau_{i}^{+}$. Therefore, $P_{o} \rightarrow 0$ as $\tau_{i+1}^{-} \rightarrow \tau_{i}^{+}$. 
Remark 1.8. The probability of immediate transaction after a transaction is zero can be shown also by the continuity of the Brownian motion.

Now we show that the expectation of the discounted value function along the sequence $\left\{\tau_{1}, \tau_{2}, \tau_{3}, \ldots \ldots\right\}$ of stopping times satisfies

Theorem 1.9. We have

$$
E\left[e^{-\delta \tau_{N+1}^{-}} \Phi\left(X\left(\tau_{N+1}^{-}\right), Y\left(\tau_{N+1}^{-}\right)\right)\right] \rightarrow 0 \text { as } N \rightarrow \infty,
$$

where $N$ is a positive integer.

Proof. Adding (1.72) and (1.73) for $i=0,1,2, \ldots, N$ and using the relation

$$
v\left(\tau_{i}^{-}\right)=\left[a \cdot X^{\gamma}\left(\tau_{i}^{-}\right)+\frac{Y^{m}\left(\tau_{i}^{-}\right)}{m}\right] \geq\left[a \cdot X^{\gamma}\left(\tau_{i}^{+}\right)+\frac{Y^{m}\left(\tau_{i}^{+}\right)}{m}\right]=v\left(\tau_{i}^{+}\right),
$$

we obtain

$$
\begin{aligned}
& e^{-\delta \tau_{N+1}^{-}} \cdot v\left(X\left(\tau_{N+1}^{-}\right), Y\left(\tau_{N+1}^{-}\right)\right) \\
= & e^{-\delta \tau_{0}^{+}} \cdot v\left(X\left(\tau_{0}^{+}\right), Y\left(\tau_{0}^{+}\right)\right) \\
& +\sum_{i=1}^{N}\left[e^{-\delta \tau_{i}^{+}} v\left(\tau_{i}^{+}\right)-e^{-\delta \tau_{i}^{-}} v\left(\tau_{i}^{-}\right)\right] \\
& +\sum_{i=0}^{N} e^{-\delta \tau_{i}^{+}} a \cdot X^{\gamma}\left(\tau_{i}^{+}\right)\left[e^{-\frac{(\delta-r \gamma)}{(1-\gamma)}\left(\tau_{i+1}^{-}-\tau_{i}^{+}\right)}-1\right] \\
& +\sum_{i=0}^{N} e^{-\delta \tau_{i}^{+}} \frac{Y^{m}\left(\tau_{i}^{+}\right)}{m}\left[e^{M_{i}}-1\right] .
\end{aligned}
$$

Taking the expectation leads to

$$
\begin{aligned}
& E\left\{e^{-\delta \tau_{N+1}^{-}} \cdot v\left(X\left(\tau_{N+1}^{-}\right), Y\left(\tau_{N+1}^{-}\right)\right)\right\} \\
& =E\left\{e^{-\delta \tau_{0}^{+}} \cdot v\left(X\left(\tau_{0}^{+}\right), Y\left(\tau_{0}^{+}\right)\right)\right\}+\sum_{i=1}^{N} E\left[e^{-\delta \tau_{i}}\left(v\left(\tau_{i}^{+}\right)-v\left(\tau_{i}^{-}\right)\right)\right] \\
& +\sum_{i=0}^{N} E\left\{e^{-\delta \tau_{i}^{+}} a \cdot X^{\gamma}\left(\tau_{i}^{+}\right)\right\}\left[e^{-\frac{(\delta-r \gamma)}{(1-\gamma)}\left(\tau_{i+1}^{-}-\tau_{i}^{+}\right)}-1\right]+\sum_{i=0}^{N} E\left\{e^{-\delta \tau_{i}^{+}} \frac{Y^{m}\left(\tau_{i}^{+}\right)}{m}\right\} \\
& \text {. }\left[E\left\{e^{M_{i}} \mid Y(t) \text { leaves or stays in NT region at } t=\tau_{N+1}^{-}\right\}-1\right] \text {. }
\end{aligned}
$$

We are assuming that a given sequence $\left\{\tau_{1}, \tau_{2}, \tau_{3}, \ldots \ldots\right\}$ satisfying $0 \leq \tau_{1}<$ $\tau_{2}<\ldots$ is a sequence of the $F_{t}$-stopping times at which the investor decides to change his portfolio. Since the investor can change the portfolio either on the transaction boundaries or inside the no-transacion region, we have 


$$
\begin{aligned}
& E\left\{e^{M_{i}} \mid Y(t) \text { leaves or stays in NT region at } t=\tau_{N+1}^{-}\right\} \\
\leq & \frac{1}{\sqrt{2 \pi}} \int_{D\left(\tau_{i}^{+}, h_{b}\left(X\left(\tau_{i+1}^{-}\right)\right)\right.}^{D\left(\tau_{i}^{+}, h_{s}\left(X\left(\tau_{i+1}^{-}\right)\right)\right.} e^{-\frac{1}{2}\left(z_{i}-m \sigma \sqrt{\tau_{i+1}^{-}-\tau_{i}^{+}}\right)^{2}} d z_{i} \\
= & \frac{1}{\sqrt{2 \pi}} \int_{G\left(\tau_{i}^{+}, h_{b}\left(X\left(\tau_{i+1}^{-}\right)\right)\right.}^{G\left(\tau_{i}^{+}, h_{s}\left(X\left(\tau_{i+1}^{-}\right)\right)\right.} e^{-\frac{1}{2} z_{i}^{2}} d z_{i},
\end{aligned}
$$

where

$$
G\left(\tau_{i}^{+}, Y\right)=\frac{\log \frac{Y}{Y\left(\tau_{i}^{+}\right)}-\left[\alpha+\left(m-\frac{1}{2}\right) \sigma^{2}\right]\left(\tau_{i+1}^{-}-\tau_{i}^{+}\right)}{\sigma \sqrt{\tau_{i+1}^{-}-\tau_{i}^{+}}} .
$$

Since $E\left\{e^{M_{i}}\right\}=1$, this implies that the last term in (1.88) is negative.

If the number of transactions is finite, there is a time $\tau_{\max }$ beyond which there is no transaction. Then, since the money decreases if there is no transaction, both the stock and money will be confined in the region given by $0 \leq X \leq$ $X\left(\tau_{\max }\right)$ and $h_{b}(X) \leq Y \leq h_{s}(X)$. Therefore, in this case

$$
\lim _{t \rightarrow \infty} e^{-\delta t} \Phi(X(t), Y(t))=0 .
$$

Now suppose we make transactions infinitely many times. Lemma 1.7 implies that there is no accumulation point in the sequence $\left\{\tau_{1}, \tau_{2}, \tau_{3}, \ldots \ldots\right\}$. Therefore, $\tau_{N}$ approaches infinity almost surely if the number of transactions are infinite. We show (1.85) by contradiction. Assume that there exists $\varepsilon>0$ such that for every $N$ there exists $i \geq N$ such that $E\left[e^{-\delta \tau_{i}^{+}} a \cdot X^{\gamma}\left(\tau_{i}^{+}\right)\right] \geq \varepsilon$ or

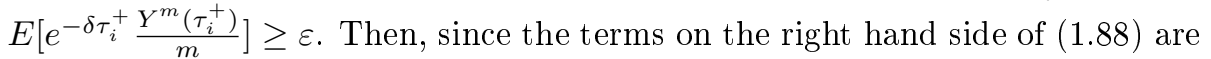
negative except the first term, it will be negative. This is a contradition since the left hand side is positive. Therefore, for every $\varepsilon>0$, there exists $N$ such

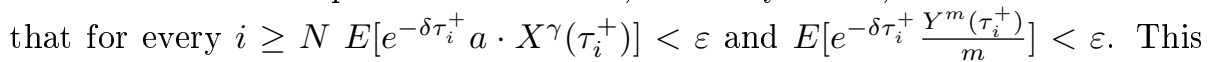
means that

$$
\lim _{i \rightarrow \infty} E\left[e^{-\delta \tau_{i}^{+}} a \cdot X^{\gamma}\left(\tau_{i}^{+}\right)\right]=\lim _{i \rightarrow \infty} E\left[e^{-\delta \tau_{i}^{+}} \frac{Y^{m}\left(\tau_{i}^{+}\right)}{m}\right]=0 .
$$

From (1.72) and (1.73)

$$
\begin{gathered}
E\left[e^{-\delta \tau_{i+1}^{-} a} \cdot X^{\gamma}\left(\tau_{i+1}^{-}\right)\right]<E\left[e^{-\delta \tau_{i}^{+}} a \cdot X^{\gamma}\left(\tau_{i}^{+}\right)\right], \\
E\left[e^{-\delta \tau_{i+1}^{-}} \frac{Y^{m}\left(\tau_{i+1}^{-}\right)}{m}\right]<E\left[e^{-\delta \tau_{i}^{+}} \frac{Y^{m}\left(\tau_{i}^{+}\right)}{m}\right] .
\end{gathered}
$$

Therefore,

$$
\lim _{N \rightarrow \infty} E\left[e^{-\delta \tau_{N+1}^{-}} \Phi\left(X\left(\tau_{N+1}^{-}\right), Y\left(\tau_{N+1}^{-}\right)\right)\right]=0 .
$$

We have shown that for every sequence $\left\{\tau_{1}, \tau_{2}, \tau_{3}, \ldots \ldots\right\}$ of stopping times which approach $\infty$, the pathwise expectation $E\left[e^{-\delta \tau_{N+1}^{-}} v\left(X\left(\tau_{N+1}^{-}\right), Y\left(\tau_{N+1}^{-}\right)\right)\right]$approaches zero. 


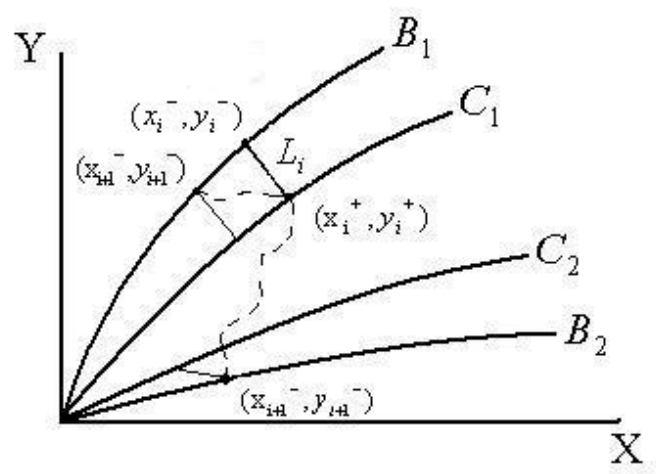

Figure 1.2: Trading details

Before we prove the verification theorem it is instructive to explain what typically happens with our formulation. Let $\left(x_{i}^{-}, y_{i}^{-}\right)$be the coordinates of the portfolio at time $\tau_{i}^{-}$on the curve $B_{1}$, where $x_{i}^{-}$and $y_{i}^{-}$represent the amount of money in the bank account and stock at time $\tau_{i}^{-}$( $i$ is a positive integer), respectively. Then we will sell the stock along the line $L_{i}$. The portfolio point will arrive the optimal point $\left(x_{0 i}^{+}, y_{0 i}^{+}\right)$, which is a point on the optimal portfolio curve $C_{1}$. Since $\left(x_{0 i}^{+}, y_{0 i}^{+}\right)$is in the NT-region, it will move to another position depending on the stochastic processes given in equations (1.1) and (1.2). From Lemma 1.5, the amount of money in the bank account decreases between any two consecutive transaction times, so the portfolio point can only move to the left. In addition, the stock follows the Geometric Brownian motion, and this will make the portfolio point either go up or go down. In comprehensive, the portfolio point will either move to the left up or move to the left down along a path of random work, until it reach either curve $B_{1}$ or curve $B_{2}$ at time $\tau_{i+1}^{-}$. If it reaches the curve $B_{1}$, we will sell the stock again by repeating the procedure provided above. If it reaches the curve $B_{2}$, we will buy the stock by using the similar ideas. The tradings for the case where $\left(x_{i}^{-}, y_{i}^{-}\right)$are the coordinates of the portfolio at time $\tau_{i}^{-}$on the curve $B_{2}$ or in the NT-region are similar. Graph that describes the movement of a portfolio point is shown in Figure 1.2.

We now verify that the solution to the HJB equation is a value function for the quasi-variational inequality problem (1.30).

Theorem 1.10. (Verification Theorem) For $v(x, y)=a x^{\gamma}+\frac{y^{m}}{m}$ define

$$
c^{*}=\left(\frac{\partial v}{\partial x}\right)^{\frac{1}{\gamma-1}}
$$

and also define the impulse control

$$
\phi^{*}:=\left(\tau_{1}^{*}, \tau_{2}^{*}, \ldots ; \xi_{1}^{*}, \xi_{2}^{*}, \ldots\right)
$$


to be the stopping times and transaction sizes with $\tau_{0}^{*}=0$ and given inductively by

$$
\begin{gathered}
\tau_{k+1}^{*}=\inf \left\{t>\tau_{k}^{*} \mid(X(t), Y(t)) \notin N T\right\}, \\
\xi_{k+1}^{*}=\xi\left(X\left(\tau_{k+1}^{*-}\right), Y\left(\tau_{k+1}^{*-}\right)\right) .
\end{gathered}
$$

Then, $\Phi$ satisfies

$$
\Phi(x, y)=\widetilde{\Phi}(0, x, y)=\sup _{c, \phi} E\left[\int_{0}^{\infty} e^{-\delta t} \frac{c^{\gamma}(x)}{\gamma} d t\right]
$$

and $w^{*}=\left(c^{*}, \phi^{*}\right)$ is optimal.

Proof. Choose $c=c^{*}$ and fix a point $q=(t, x, y)$. For $i=0,1,2, \ldots, N$, by the Itô-Doeblin formula,

$$
\begin{aligned}
& e^{-\delta \tau_{i+1}^{*-}} v\left(X\left(\tau_{i+1}^{*-}\right), Y\left(\tau_{i+1}^{*-}\right)\right) \\
= & e^{-\delta \tau_{i}^{*}} v\left(X\left(\tau_{i}^{*}\right), Y\left(\tau_{i}^{*}\right)\right)+\int_{\tau_{i}^{*}}^{\tau_{i+1}^{*}} e^{-\delta t} L^{c^{*}} v(X(t), Y(t)) d t \\
& +\int_{\tau_{i}^{*}}^{\tau_{i+1}^{*}} e^{-\delta t} \sigma Y(t) d W(t),
\end{aligned}
$$

where

$$
L^{c^{*}} v(x, y)=-\delta v+\left(r x-c^{*}\right) \frac{\partial v}{\partial x}+\alpha y \frac{\partial v}{\partial y}+\frac{1}{2} \sigma^{2} y^{2} \frac{\partial^{2} v}{\partial y^{2}}
$$

Since

$$
e^{-\delta t} L^{c^{*}} v(X(t), Y(t))=-e^{-\delta t} \frac{c^{* \gamma}(X(t))}{\gamma},
$$

substituting this in (1.101) and rearranging the terms, we have

$$
\begin{aligned}
& e^{-\delta \tau_{i}^{*}} v\left(X\left(\tau_{i}^{*}\right), Y\left(\tau_{i}^{*}\right)\right) \\
= & e^{-\delta \tau_{i+1}^{*-}} v\left(X\left(\tau_{i+1}^{*-}\right), Y\left(\tau_{i+1}^{*-}\right)\right)+\int_{\tau_{i}^{*}}^{\tau_{i+1}^{*}} e^{-\delta t} \frac{c^{* \gamma}(X(t))}{\gamma} d t \\
& -\int_{\tau_{i}^{*}}^{\tau_{i+1}^{*}} e^{-\delta t} \sigma Y(t) d W(t) .
\end{aligned}
$$

Summering (1.104) for $i=0$ to $i=N$ and taking the expectation, we obtain

$$
\begin{aligned}
& v(x, y)+\sum_{i=1}^{N} E\left[e^{-\delta \tau_{i}^{*}} v\left(X\left(\tau_{i}^{*}\right), Y\left(\tau_{i}^{*}\right)\right)-e^{-\delta \tau_{i}^{*-}} v\left(X\left(\tau_{i}^{*}-\right), Y\left(\tau_{i}^{*}-\right)\right)\right] \\
= & E\left[e^{-\delta \tau_{N+1}^{*-}} v\left(X\left(\tau_{N+1}^{*-}\right), Y\left(\tau_{N+1}^{*-}\right)\right)+E\left[\int_{0}^{\tau_{N+1}^{*}} e^{-\delta t} \frac{c^{* \gamma}(X(t))}{\gamma} d t\right] .(1.105)\right.
\end{aligned}
$$


If the investor make transactions on the transaction boundaries,

$$
v\left(X\left(\tau_{i}^{*}\right), Y\left(\tau_{i}^{*}\right)\right)=M v\left(X\left(\tau_{i}^{*}{ }^{-}\right), X\left(\tau_{i}^{*}-\right)\right) .
$$

Therefore, we obtain

$$
v(x, y)=E\left[e^{-\delta \tau_{N+1}^{*-}} v\left(X\left(\tau_{N+1}^{*-}\right), Y\left(\tau_{N+1}^{*-}\right)\right)+E\left[\int_{0}^{\tau_{N+1}^{*}} e^{-\delta t} \frac{c^{* \gamma}(X(t))}{\gamma} d t\right] .\right.
$$

Let $N \rightarrow \infty$ and use Theorem 1.9. Then, we have

$$
\Phi(x, y)=E\left[\int_{0}^{\infty} e^{-\delta t} \frac{c^{* \gamma}(x)}{\gamma} d t\right]
$$

and this gives us

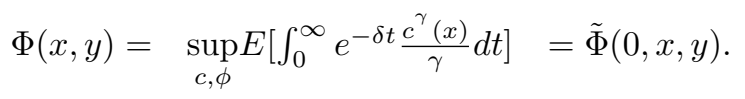

Thus, (1.100) satisfies for all $(x, y) \in S_{+}$and $w^{*}$ is optimal.

Remark 1.11. Usually, the condition

$$
\lim _{N \rightarrow \infty} E\left[e^{-\delta \tau_{N+1}^{-}} v\left(X\left(\tau_{N+1}^{-}\right), Y\left(\tau_{N+1}^{-}\right)\right)\right]=0
$$

is assumed to hold or given as the terminal condition.

\subsection{Viscosity Solutions}

It has been shown that the value function $\Phi(x, y)=\sup _{c, \phi} E\left[\int_{0}^{\infty} e^{-\delta t} \frac{c^{\gamma}(t)}{\gamma} d t\right]$ is a viscosity solution [23, 24]. Since we know that (1.18) in Theorem 1.1 is an explicit solution to the HJB equation and we define the value function $\Phi$ which is given in (1.29), it is interesting to prove that $\Phi$ is a viscosity solution for the quasi-variational inequality problem (1.30).

Definition 1.12. Suppose $G$ is a topological space, $x_{0}$ is a point in $G$ and $\psi: G \rightarrow R$ is a function.

(1)We say that $\varphi$ is upper semi-continuous (usc) at $x_{0}$ if $\limsup _{x \rightarrow x_{0}} \varphi(x) \leq \varphi\left(x_{0}\right)$. The function $\varphi$ is called upper semi-continuous if it is upper semi-continuous at every point of $G$.

(2) Similarly, we say that $\varphi$ is lower semi-continuous (lsc) at $x_{0}$ if $\liminf _{x \rightarrow x_{0}} \varphi(x) \geq$ $\varphi\left(x_{0}\right)$. The function $\varphi$ is called lower semi-continuous if it is lower semicontinuous at every point of $G$.

(3) We let $U S C(G)$ and $L S C(G)$ denote the set of usc functions and $l s c$ functions on $G$, respectively. 
Definition 1.13. Let $S_{+}^{0}$ be the interior of $S_{+}$and $\zeta=(x, y)$. We define $F$ as follows.

$$
\begin{aligned}
& F\left(D^{2} v(\zeta), D v(\zeta), v(\zeta), \zeta\right) \\
& \left\{\begin{array}{c}
\max _{c, \phi}\left\{-\delta v+(r x-c) \frac{\partial v}{\partial x}+\alpha y \frac{\partial v}{\partial y}+\frac{1}{2} \sigma^{2} y^{2} \frac{\partial^{2} v}{\partial y^{2}}+\frac{c^{\gamma}}{\gamma}\right. \\
, M v-\Phi\} \text { if } \zeta \in S_{+}^{0} \\
\max _{c, \phi}\left\{-\delta v+(r x-c) \frac{\partial v}{\partial x}+\frac{c^{\gamma}}{\gamma}, M v-\Phi\right\} \\
\quad \text { if } y=0 \text { and } x \in(0, \infty), \\
\max _{\phi}\left\{-\delta v+\alpha y \frac{\partial v}{\partial y}+\frac{1}{2} \sigma^{2} y^{2} \frac{\partial^{2} v}{\partial y^{2}}, M v-\Phi\right\} \\
\text { if } x=0 \text { and } y \in(0, \infty) .
\end{array}\right.
\end{aligned}
$$

(1) A function $\psi \in U S C\left(S_{+}\right)$is a viscosity subsolution of

$$
F\left(D^{2} v(\zeta), D v(\zeta), v(\zeta), \zeta\right)=0
$$

if for every $\zeta_{0} \in S_{+}$and for every function $f \in C^{2}\left(S_{+}\right)$satisfying $f \geq \psi$ on $S_{+}$ and $f\left(\zeta_{0}\right)=\psi\left(\zeta_{0}\right)$, we have

$$
F\left(D^{2} f\left(\zeta_{0}\right), D f\left(\zeta_{0}\right), f\left(\zeta_{0}\right), \zeta_{0}\right) \geq 0 .
$$

(2) A function $\psi \in L S C\left(S_{+}\right)$is a viscosity supersolution of (1.111) if for every $\zeta_{0} \in S_{+}$and for every function $f \in C^{2}\left(S_{+}\right)$satisfying $f \leq \psi$ on $S_{+}$and $f\left(\zeta_{0}\right)=\psi\left(\zeta_{0}\right)$, we have

$$
F\left(D^{2} f\left(\zeta_{0}\right), D f\left(\zeta_{0}\right), f\left(\zeta_{0}\right), \zeta_{0}\right) \leq 0 .
$$

(3) A function $\psi: S_{+} \rightarrow R$ is a viscosity solution of (1.111) if $\psi$ is locally bounded and it is a viscosity sub and super solution of (1.111).

Theorem 1.14. The value function $\Phi$ is a viscosity solution of (1.111).

Proof. (1) For $\zeta \in S_{+}^{0}$, we prove that $\Phi(x, y)=\max [v(x, y), M v(x, y)]$ is a viscosity subsolution of the first case in (1.110). It is easy to see that $v$ is $C^{2}$. Consider $f$ be a $C^{2}$ function in a neighborhood of $S_{+}^{0}$ and let $\zeta_{0}=\left(x_{0}, y_{0}\right) \in S_{+}^{0}$ be such that $f \geq \Phi$ on $S_{+}^{0}$ and $f\left(\zeta_{0}\right)=\Phi\left(\zeta_{0}\right)$. We show two cases separately.

Case (i). $v\left(\zeta_{0}\right) \leq M v\left(\zeta_{0}\right)$. Then, $f\left(\zeta_{0}\right)=\Phi\left(\zeta_{0}\right)=\max \left[v\left(\zeta_{0}\right), M v\left(\zeta_{0}\right)\right]=$ $M v\left(\zeta_{0}\right)$. Since $M v\left(\zeta_{0}\right)=v\left(x^{\prime}, y^{\prime}\right) \leq f\left(x^{\prime}, y^{\prime}\right) \leq M f\left(\zeta_{0}\right)$, we have

$$
\begin{aligned}
& \max _{c, \phi}\left\{\left[-\delta f+(r x-c) \frac{\partial f}{\partial x}+\alpha y \frac{\partial f}{\partial y}+\frac{1}{2} \sigma^{2} y^{2} \frac{\partial^{2} f}{\partial y^{2}}+\frac{c^{\gamma}}{\gamma}\right]\left(\zeta_{0}\right),\right. \\
& {\left.[M f-\max (M f, f)]\left(\zeta_{0}\right)\right\} } \\
\geq & {[M f-\max (M f, f)]\left(\zeta_{0}\right) } \\
= & {[M f-\max (M f, M v)]\left(\zeta_{0}\right) \geq 0 . }
\end{aligned}
$$

So (1.112) holds. 
Case (ii). $v\left(\zeta_{0}\right)>M v\left(\zeta_{0}\right)$. We need to prove

$$
\max _{c}\left[-\delta f+(r x-c) \frac{\partial f}{\partial x}+\alpha y \frac{\partial f}{\partial y}+\frac{1}{2} \sigma^{2} y^{2} \frac{\partial^{2} f}{\partial y^{2}}+\frac{c^{\gamma}}{\gamma}\right]\left(\zeta_{0}\right) \geq 0 .
$$

Since $f \geq \Phi \geq v$ on $S_{+}^{0}$ and $f\left(\zeta_{0}\right)=\Phi\left(\zeta_{0}\right)=v\left(\zeta_{0}\right), f-v$ has a local minimum at $\zeta_{0}$. Therefore, $\frac{\partial f}{\partial x}\left(\zeta_{0}\right)=\frac{\partial v}{\partial x}\left(\zeta_{0}\right), \frac{\partial f}{\partial y}\left(\zeta_{0}\right)=\frac{\partial v}{\partial y}\left(\zeta_{0}\right)$ and $\frac{\partial^{2} f}{\partial y^{2}}\left(\zeta_{0}\right) \geq \frac{\partial^{2} v}{\partial y^{2}}\left(\zeta_{0}\right)$ [7]. This implies

$$
\begin{array}{r}
\max _{c}\left[-\delta f+(r x-c) \frac{\partial f}{\partial x}+\alpha y \frac{\partial f}{\partial y}+\frac{1}{2} \sigma^{2} y^{2} \frac{\partial^{2} f}{\partial y^{2}}+\frac{c^{\gamma}}{\gamma}\right]\left(\zeta_{0}\right) \\
\geq \max _{c}\left[-\delta v+(r x-c) \frac{\partial v}{\partial x}+\alpha y \frac{\partial v}{\partial y}+\frac{1}{2} \sigma^{2} y^{2} \frac{\partial^{2} v}{\partial y^{2}}+\frac{c^{\gamma}}{\gamma}\right]\left(\zeta_{0}\right) .
\end{array}
$$

We choose $c^{*}=\left(\frac{\partial v}{\partial x}\right)^{\frac{1}{\gamma-1}}$ and substitute $v(x, y)=a x^{\gamma}+\frac{y^{m}}{m}$ into the right hand side of (1.116). Then, by Theorem 1.1 we see that the right hand side of (1.116) is zero.

The proofs for the cases where $x=0$ or $y=0$ are similar.

(2) For $\zeta \in S_{+}^{0}$, we prove that $\Phi(x, y)=\max [v(x, y), M v(x, y)]$ is a viscosity supersolution of the first case in (1.110). So we let $f$ be a $C^{2}$ function in a neighborhood of $S_{+}^{0}$ and we let $\zeta_{0} \in S_{+}^{0}$ such that $f \leq \Phi=\max (v, M v)$ on $S_{+}^{0}$ and $f\left(\zeta_{0}\right)=\Phi\left(\zeta_{0}\right)=\max \left[v\left(\zeta_{0}\right), M v\left(\zeta_{0}\right)\right]$. We want to show (1.113).

Since $[M f-\max (M f, f)]\left(\zeta_{0}\right) \leq 0$, we only need to prove

$$
\max _{c}\left[-\delta f+(r x-c) \frac{\partial f}{\partial x}+\alpha y \frac{\partial f}{\partial y}+\frac{1}{2} \sigma^{2} y^{2} \frac{\partial^{2} f}{\partial y^{2}}+\frac{c^{\gamma}}{\gamma}\right]\left(\zeta_{0}\right) \leq 0 .
$$

For $\varepsilon>0$ and $\widehat{w}=\widehat{w}_{\varepsilon, c}$ be an admissible control begining with a constant consumption rate $c>0$ and no transactions up to the first time $\tau_{\varepsilon}$ at which the stochastic process exists from

$$
K_{\varepsilon}=\left\{(s, x, y) ;\left|(s, x, y)-\left(0, x_{0}, y_{0}\right)\right|<\varepsilon\right\} \cap \widetilde{S}_{+},
$$

where $\zeta_{0}=\left(x_{0}, y_{0}\right)$ and $\widetilde{S}_{+}=R^{+} \times S_{+}$. Choose $\zeta_{n} \in K_{\varepsilon}$ such that $\zeta_{n} \rightarrow \zeta_{0}$ and $\Phi\left(\zeta_{n}\right) \rightarrow \Phi\left(\zeta_{0}\right)$ as $n \rightarrow \infty$. By using It $\widehat{o}-$ Doeblin formula and the definition of expectation, we obtain

$$
\begin{gathered}
\Phi\left(\zeta_{n}\right) \geq E^{0, \zeta_{n}}\left[e^{-\delta \tau_{\varepsilon}} \Phi\left(X^{\widehat{w}}\left(\tau_{\varepsilon}\right), Y^{\widehat{w}}\left(\tau_{\varepsilon}\right)\right)\right]+E^{0, \zeta_{n}}\left[\int_{0}^{\tau_{\varepsilon}} e^{-\delta t} \frac{c^{\gamma}(X(t))}{\gamma} d t\right] \\
\geq E^{0, \zeta_{n}}\left[e^{-\delta \tau_{\varepsilon}} f\left(X^{\widehat{w}}\left(\tau_{\varepsilon}\right), Y^{\widehat{w}}\left(\tau_{\varepsilon}\right)\right)\right]+E^{0, \zeta_{n}}\left[\int_{0}^{\tau_{\varepsilon}} e^{-\delta t} \frac{c^{\gamma}(X(t))}{\gamma} d t\right] \\
=f\left(\zeta_{n}\right)+E^{0, \zeta_{n}}\left[\int_{0}^{\tau_{\varepsilon}} e^{-\delta t} L^{c} f\left(X^{\widehat{w}}(t), Y^{\widehat{w}}(t)\right) d t\right]+E^{0, \zeta_{n}}\left[\int_{0}^{\tau_{\varepsilon}} e^{-\delta t} \frac{c^{\gamma}(X(t))}{\gamma} d t\right]
\end{gathered}
$$


where $L^{c} f(x, y)=-\delta f+(r x-c) \frac{\partial f}{\partial x}+\alpha y \frac{\partial f}{\partial y}+\frac{1}{2} \sigma^{2} y^{2} \frac{\partial^{2} f}{\partial y^{2}}$ and for all $n$. From (1.119),

$$
E^{0, \zeta_{n}}\left[\int_{0}^{\tau_{\varepsilon}} e^{-\delta t}\left\{L^{c} f\left(X^{\widehat{w}}(t), Y^{\widehat{w}}(t)\right)+\frac{c^{\gamma}(X(t))}{\gamma}\right\} d t\right] \leq \Phi\left(\zeta_{n}\right)-f\left(\zeta_{n}\right)
$$

for all $n$. Taking the limit as $n \rightarrow \infty$, we obtain

$$
E^{0, \zeta_{0}}\left[\int_{0}^{\tau_{\varepsilon}} H(t) d t\right] \leq 0
$$

where

$$
H(t)=e^{-\delta t}\left(L^{c} f\left(X^{\widehat{w}}(t), Y^{\widehat{w}}(t)\right)+\frac{c^{\gamma}(X(t))}{\gamma}\right) .
$$

By dividing the left-hand side by $E^{0, \zeta_{0}}\left[\tau_{\varepsilon}\right]$ we get

$$
\frac{E^{0, \zeta_{0}}\left[\int_{0}^{\tau_{\varepsilon}} H(t) d t\right]}{E^{0, \zeta_{0}}\left[\tau_{\varepsilon}\right]}=\frac{E^{0, \zeta_{0}}\left[\int_{0}^{\tau_{\varepsilon}}[H(t)-H(0)] d t\right]+H(0) E^{0, \zeta_{0}}\left[\tau_{\varepsilon}\right]}{E^{0, \zeta_{0}}\left[\tau_{\varepsilon}\right]} \rightarrow H(0)
$$

as $\varepsilon \rightarrow 0$, since $H(t)$ is continous at $t=0$. Thus, we have

$$
H(0)=\left[-\delta f+(r x-c) \frac{\partial f}{\partial x}+\alpha y \frac{\partial f}{\partial y}+\frac{1}{2} \sigma^{2} y^{2} \frac{\partial^{2} f}{\partial y^{2}}+\frac{c^{\gamma}}{\gamma}\right]\left(\zeta_{0}\right) \leq 0
$$

for all $c \geq 0$ such that $\widehat{w}_{\varepsilon, c}$ is admissible for $\varepsilon$ small enough [20].

The proofs for the cases where $x=0$ or $y=0$ are similar.

(3) (1) and (2) imply that $\Phi$ is a viscosity solution of (1.111).

\section{$1.7 \quad$ Numerical results}

In this section we examine numerically what we discussed in the previous sections. One goal is to find numerically the transaction and no-transaction regions as well as the optimal portfolio and transaction boundaries for various cases of the parameters. Another goal is to compare the approximate transaction boundaries obtained in Section 1.4 and the one obtained numerically.

As the parameters we consider $\gamma, m$ and $k, \lambda$. The parameters $\gamma$ and $m$ represent the investors preference to safe and risky assets. We observed that $\gamma$ and $m$ affect the convexity and concavity of the optimal portfolio and transaction curves. If $\gamma>m$, the graphs of the optimal portfolio curves are concave in the $x y$-plane. This shows that as the wealth $x+y$ increases the investor allocates more wealth to the safe asset, indicating that the case where $\gamma>m$ is appropriate for conservative investors. On the other hand, if $\gamma<m$, the graphs of the optimal portfolio curves are convex in the $x y$-plane. This shows that as the wealth $x+y$ increases the investor allocates more wealth to the risky asset, indicating that the case where $\gamma<m$ is appropriate for risk-taking or entrepreneur investors. In Subsection 1.7.1 we examine the case where $\gamma>m$. 

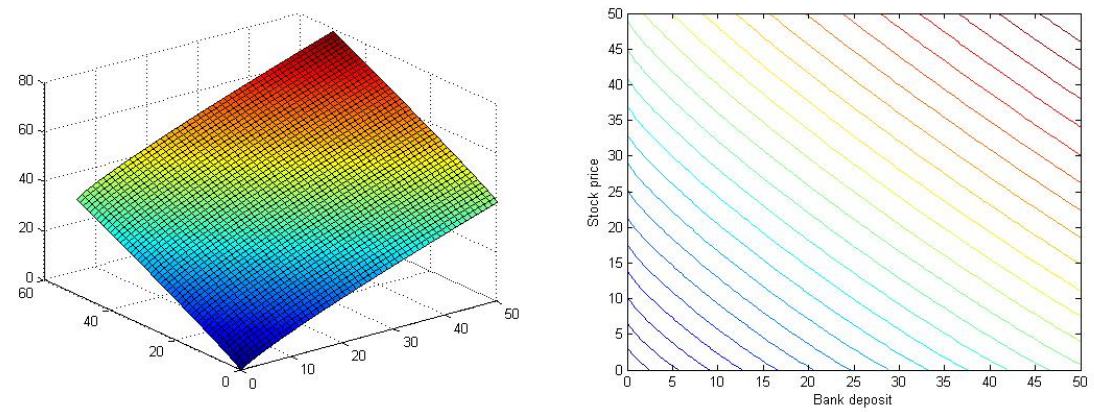

Figure 1.3: (Left) The value function $v(x, y)=a x^{\gamma}+\frac{y^{m}}{m}$. (Right) The contour curves for the value function $v(x, y)=a x^{\gamma}+\frac{y^{m}}{m}$.

The graphs and explanations of the four different cases of $k$ and $\lambda$ are presented. The case where $\gamma<m$ will be discussed in Subsection 1.7.2.

Concerning the approximate transaction boundaries, in Subsection 1.7.3 we examine how the approximation depends on $k$ and $\left(x_{0}, y_{0}\right)$. We observe that the approximations are good for smaller values of $k$.

\subsubsection{The case when $\gamma>m$.}

For the numerical calculation, we choose $\lambda=0.1, k=0.03$ if $(k \neq 0), \alpha=0.11$, $\sigma=0.25, r=0.07, \delta=0.09, \gamma=0.90$. Then, $m$ is given by $m=0.8537$. In this case the allocation of wealth to the risky asset decreases as the wealth increases. This case may represent a conservative investor who would allocate the smaller portion of his wealth to the risky asset as his wealth increases. The graphs of the value function $v(x, y)=a x^{\gamma}+\frac{y^{m}}{m}$ and its contour curves are given in Figure 1.3. We use Matlab for programming. For each given $x$ and $y$ which represent the amount of money in the bank account and stock, we use the Matlab function "fminbnd" to compute the transaction size $\xi$ so that the value function attains the maximum value along the line of trading. Then compare the values of $v(x, y)$ and $v\left(x^{\prime}, y^{\prime}\right)=M v(x, y)$, where $M$ is the intervention operator defined in (2.47). If $v\left(x^{\prime}, y^{\prime}\right) \geq v(x, y)$, we trade the stock. Otherwise, we do not trade. Depending on the values of $k$ and $\lambda$, we discuss the problem (1.30) numerically in Subsections 1.7.1.1 to 1.7.1.3.

\subsubsection{The case when $k>0, \lambda>0$.}

This is the case where both fixed and proportional transaction costs are present. We have $x^{\prime}=x-k-\xi-\lambda \xi$ if $\xi>0$ and $x^{\prime}=x-k-\xi+\lambda \xi$ if $\xi<0$. We first explain various notations in Figure 1.4. It is easily seen that the first quadrant has been divided into three regions. The shaded region in the left side is the sell region, the shaded region in the right side is the buy region, and the middle region, which is bounded between the green curve and red curve, 
is the no-transaction (NT) region. Suppose $\left(x_{1}, y_{1}\right)$ is the current position for the portfolio in the sell region, where $x_{1}$ and $y_{1}$ represent the amount of money in the bank account and stock, respectively. Then, the line $L_{1}$ starting from $\left(x_{1}, y_{1}\right)$ is the line along which the stock is exchanged to the money and is given by

$$
x_{01}=x_{1}-k-\xi+\lambda \xi, y_{01}=y_{1}+\xi, \xi<0,
$$

or

$$
x_{01}+(1-\lambda) y_{01}=x_{1}+(1-\lambda) y_{1}-k, x_{01}>x_{1} .
$$

The distance between $\left(x_{1}, y_{1}\right)$ and $P_{1}\left(x_{01}, y_{01}\right)$ is the transaction size $\left|\xi_{1}\right|$, i.e., we need to sell $\left|\xi_{1}\right|\left(\xi_{1}<0\right)$ shares of stock. Similarly, suppose $\left(x_{2}, y_{2}\right)$ is the current position in the buy region. Then, the line $L_{2}$ is the line starting from $\left(x_{2}, y_{2}\right)$ along which the money is exchanged to the stock and is given by

$$
x_{02}=x_{2}-k-\xi-\lambda \xi, y_{02}=y_{2}+\xi, \xi>0,
$$

or

$$
x_{02}+(1+\lambda) y_{02}=x_{2}+(1+\lambda) y_{2}-k, x_{02}<x_{2} .
$$

The distance between $\left(x_{2}, y_{2}\right)$ and $P_{2}\left(x_{02}, y_{02}\right)$ is the transaction size $\left|\xi_{2}\right|$, i.e., we need to buy $\left|\xi_{2}\right|\left(\xi_{2}>0\right)$ shares of stock.

As explained in Section 1.4 the optimal portfolio curve $C_{1}$ is the set of $\left(x_{01}, y_{01}\right)$ at which the value function attains its maximum along the line $L_{1}$ for each $\left(x_{1}, y_{1}\right)$ given in the sell region. Similarly, the optimal portfolio curve $C_{2}$ is the set of $\left(x_{02}, y_{02}\right)$ at which the value function attains its maximum along the line $L_{2}$ for each $\left(x_{2}, y_{2}\right)$ given in the buy region.

The curve $B_{1}$ is the transaction boundary between the sell and no-transaction region and in Figure 1.4 it is the boundary between the left shaded region and the middle region. The approximate curve for $B_{1}$ obtained in Theorem 1.3 is the green line in Figure 1.4. Similarly, the curve $B_{2}$ is the transaction boundary between the buy and no-transaction region and in Figure 1.4 it is the boundary between the right shaded region and the middle region. The approximate curve for $B_{2}$ obtained in Theorem 1.3 is the red line in Figure 1.4. For small values of $k$ the approximate transaction boundaries agree well with the boundary curves $B_{1}$ and $B_{2}$ obtained numerically.

\subsubsection{The case when $k>0, \lambda=0$.}

This is the case when there is a fixed transaction cost $k>0$ after each trades, but without proportional transaction costs, i.e. $\lambda=0$. In Figure 1.5 the curve $C_{1}$ (or $C_{2}$ ) gives the curve of the optimal portfolio for a given wealth level $\left(x_{0}, y_{0}\right)$ on the sell (or buy) region, and the blank region in the first quadrant is the NT region. Compare Figure 1.5 with Figure 1.4. We find that the curves of the optimal portfolios $C_{1}$ and $C_{2}$ overlap as we see the curves in (1.33) and (1.34) are identical if $\lambda=0$. 


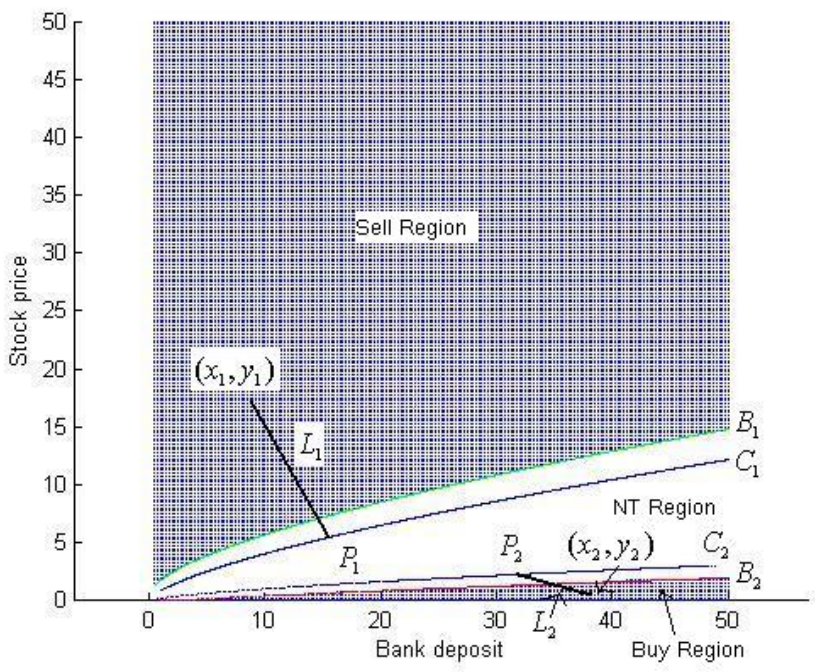

Figure 1.4: Transaction regions and NT region when $k>0, \lambda>0$ and $m=$ 0.8537 .

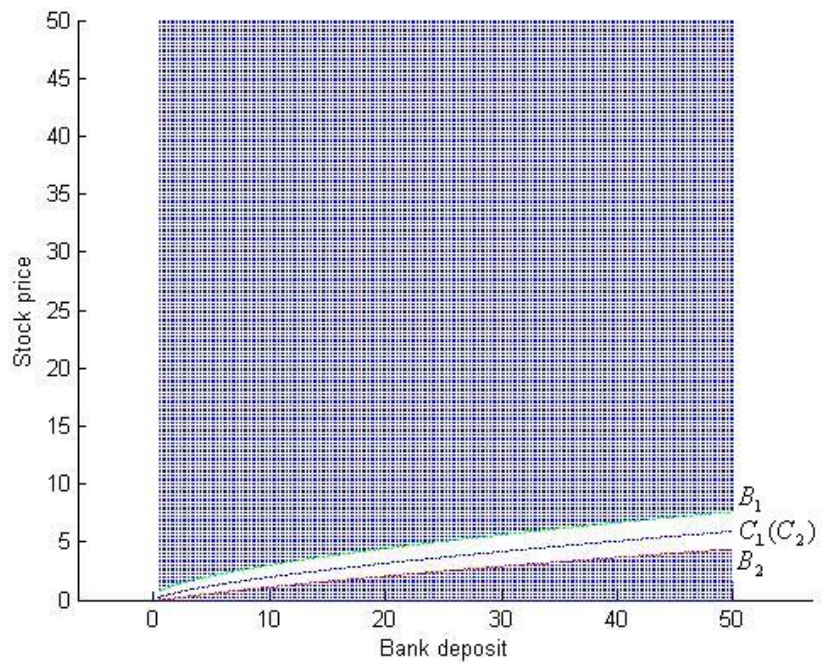

Figure 1.5: Transaction regions and NT region when $k>0, \lambda=0$ and $m=$ 0.8537 . 


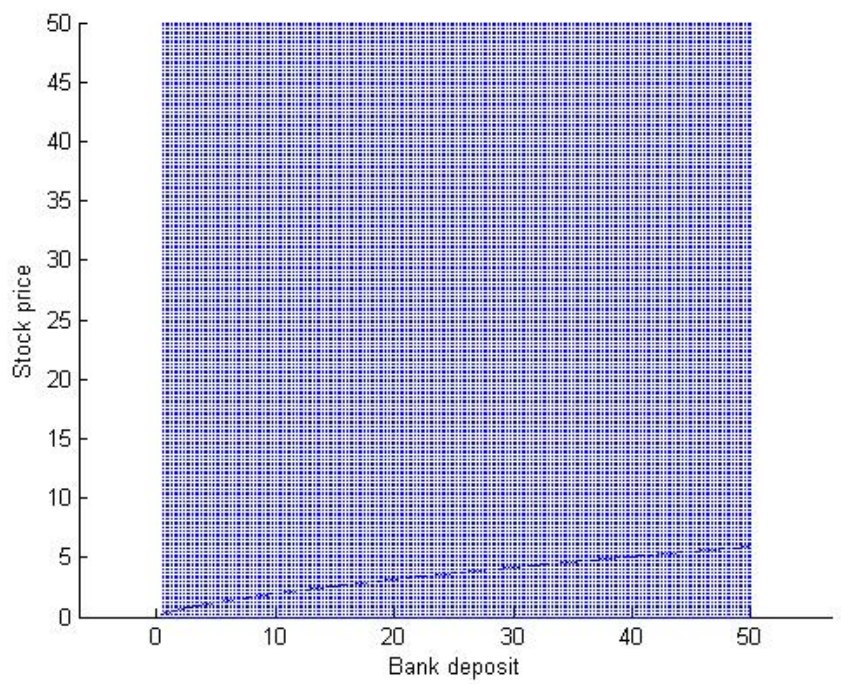

Figure 1.6: Transaction regions and NT region when $k=0, \lambda=0, m=0.8537$.

\subsubsection{The cases when $k=0$.}

The cases when $k=0$ are for the sake of comparison with the cases when $k>0$. Graphs when $k=0$ are shown in Figure 1.6 and 1.7. There are two cases. In Figure 1.6, the case where both $k$ and $\lambda$ are zero are treated. The curves $C_{1}$ and $C_{2}$ overlap and they give the allocation in the optimal portfolio for a given wealth level $w=x+y$. They play the similar role to the "Merton line" introduced in Davis and Norman [8]. We should note that in the Merton's formulation the consumption is a function of wealth and in the current formulation it is a function of money. Also, in the Merton's formulation the independent variable is the wealth and the relative weight of the money and the risky asset is a control variable. On the other hand both the money and the risky asset are independent variables in this formulation. These differences in the formulation may be the reasons why "Merton line" is no longer a straight line in the current formulation.

If $\lambda>0, C_{1}$ and $C_{2}$ no longer overlap. On the other hand, they coincide with the transaction boundaries.

\subsubsection{The case when $m>\gamma$.}

As an example we choose $\lambda=0.1, k=0.03$ (if $k \neq 0$ ), $\alpha=0.11, \sigma=0.28$, $r=0.07, \delta=0.1, \gamma=0.9$, then $m=0.9318$. In this case the allocation of wealth to the risky asset increases as the wealth increases. This case may represent a risk-taking investor who is willing to allocate the larger portion of his wealth to 


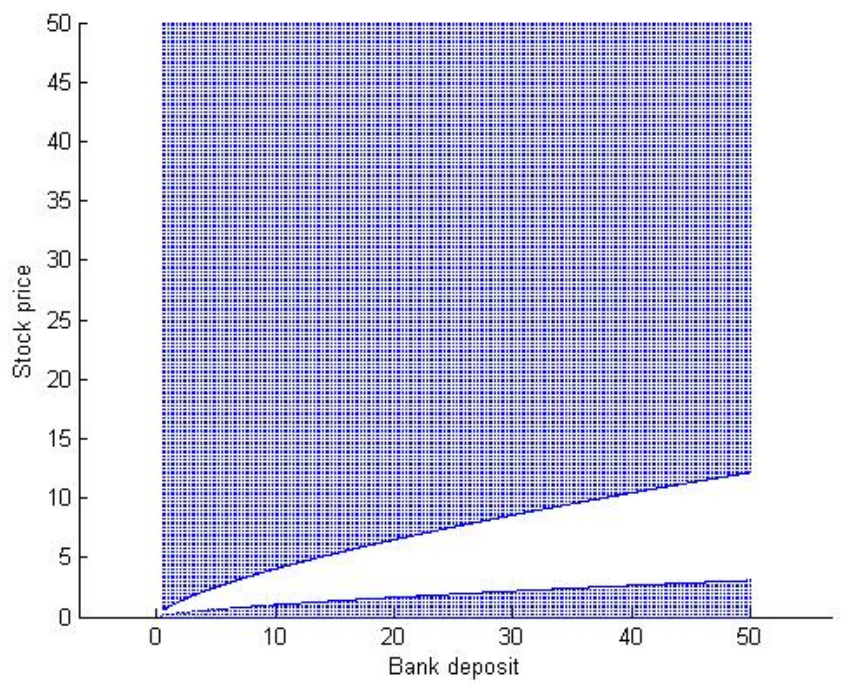

Figure 1.7: Transaction regions and NT region when $k=0, \lambda>0, m=0.8537$.

the risky asset as his wealth increases. The case when $k>0, \lambda=0$ is shown in Figures 1.8 and the case when $k>0, \lambda>0$ is shown in Figure 1.9. $B_{1}$ (or $B_{2}$ ) is the boundary between the sell region (or buy region) and NT region. The curve in the red (or green) is the approximate boundary. For a small $k$ the approximate transaction boundary and the corresponding numerical boundary are in good agreement. We see clearly that all of the curves $C_{1}, C_{2}, B_{1}$ and $B_{2}$ are concave upward curves.

\subsubsection{The approximate transaction boundaries}

We take the first three terms in the power series approximations obtained in Theorem 1.3 and examine the effects of $k$ in the approximation. We use the first three terms in the power of $k$, i.e.,

$$
x=x_{0}+\sum_{n=1}^{2} a_{n} k^{n / 2}, \quad y=y_{0}+\sum_{n=1}^{2} b_{n} k^{n / 2},
$$

where the coefficients $a_{i}$ and $b_{i}(i=1,2)$ are given in (1.47) and (1.50). Except for $k$ we use the same parameters as in Subsection 1.7.2. The points $(\widehat{x}, \widehat{y})$ on the transaction curve $B_{1}$ depend on the fixed transaction cost $k$ and the points $\left(x_{0}, y_{0}\right)$ on the optimal portfolio curve $C_{1}$. Similar situation applies for the points on the transaction curve $B_{2}$. The results are reported in Figures 1.10 to 1.12. We observe that the curve $B_{1}$ (or $B_{2}$ ) separates away from curve $C_{1}$ (or $C_{2}$ ) as $k$ increases. We also see that both the sell and buy regions recede as 


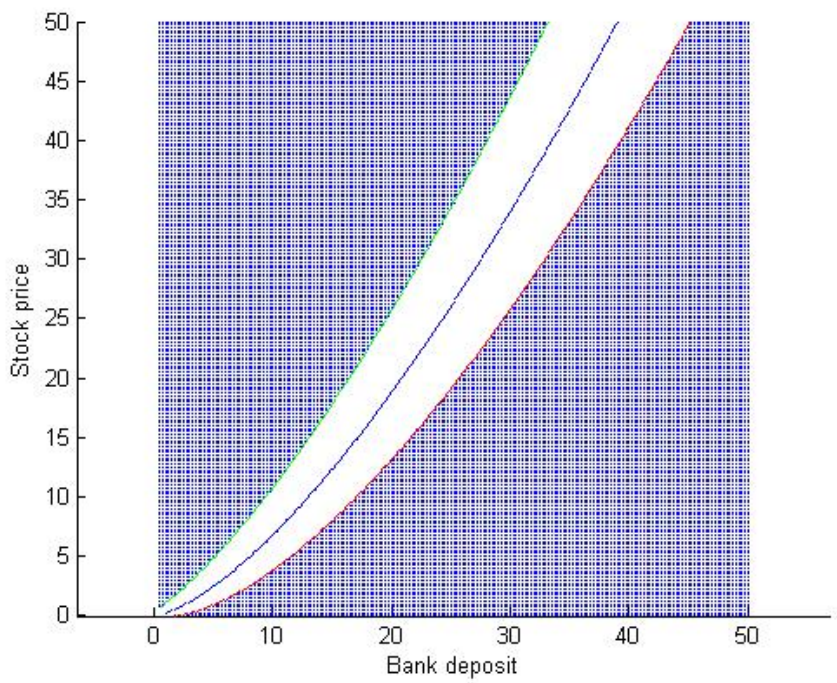

Figure 1.8: Transaction regions and NT region when $k>0, \lambda=0$ and $m=$ 0.9318 .

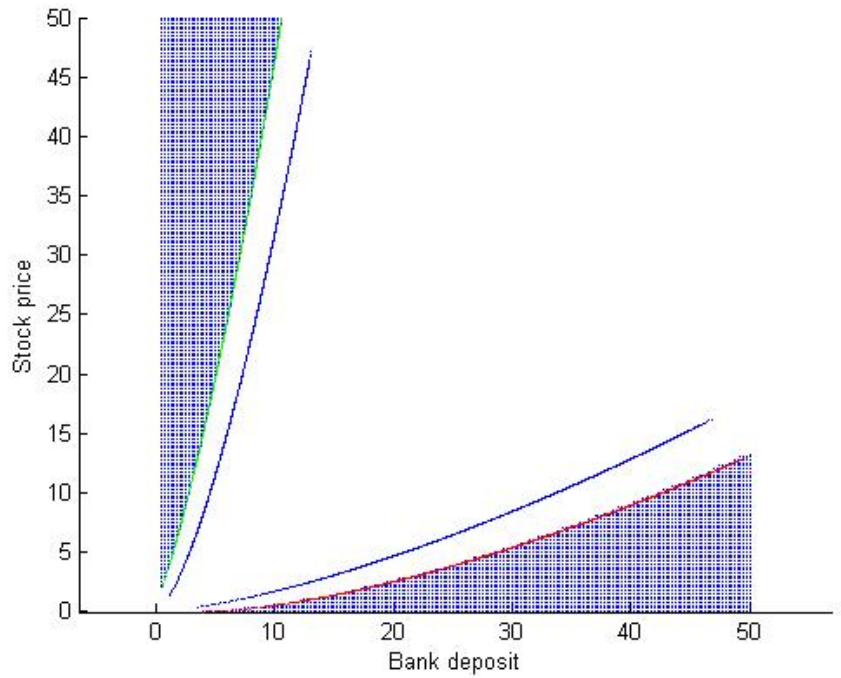

Figure 1.9: Transaction regions and NT region when $k>0, \lambda>0$ and $m=$ 0.9318 


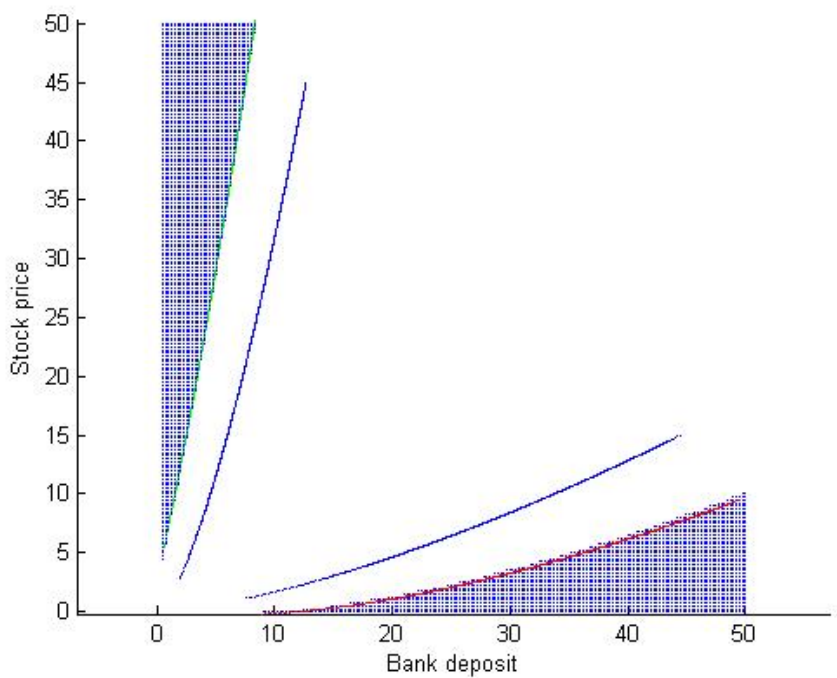

Figure 1.10: Transaction regions and NT region when $k=0.1, \lambda>0$ and $m=0.9318$.

$k$ increases. The approximations (1.128) deteriorate as $k$ increases because we take only three terms for the approximations.

\subsection{Explicit solutions for exponential and natural logarithm utility functions}

\subsubsection{Exponential utility function}

Theorem 1.15. Suppose that the utility function is an exponential function given by

$$
u(c)=1-e^{-\gamma c},
$$

where $\gamma>0$, then an explicit solution for the value function which satisfies

$$
\max _{c}\left[-\delta v+(r x-c) \frac{\partial v}{\partial x}+\alpha y \frac{\partial v}{\partial y}+\frac{1}{2} \sigma^{2} y^{2} \frac{\partial^{2} v}{\partial y^{2}}+u(c)\right]=0
$$

is given by

$$
v(x, y)=-\frac{1}{r} e^{1-\frac{\delta}{r}-r \gamma x}+\frac{y^{m}}{m}+\frac{1}{\delta} .
$$

Proof. Differentiating (1.130) with respect to $c$ and solving for $c$, we have

$$
c^{*}=-\frac{1}{\gamma} \ln \left(\frac{1}{\gamma} \frac{\partial v}{\partial x}\right) \text {. }
$$




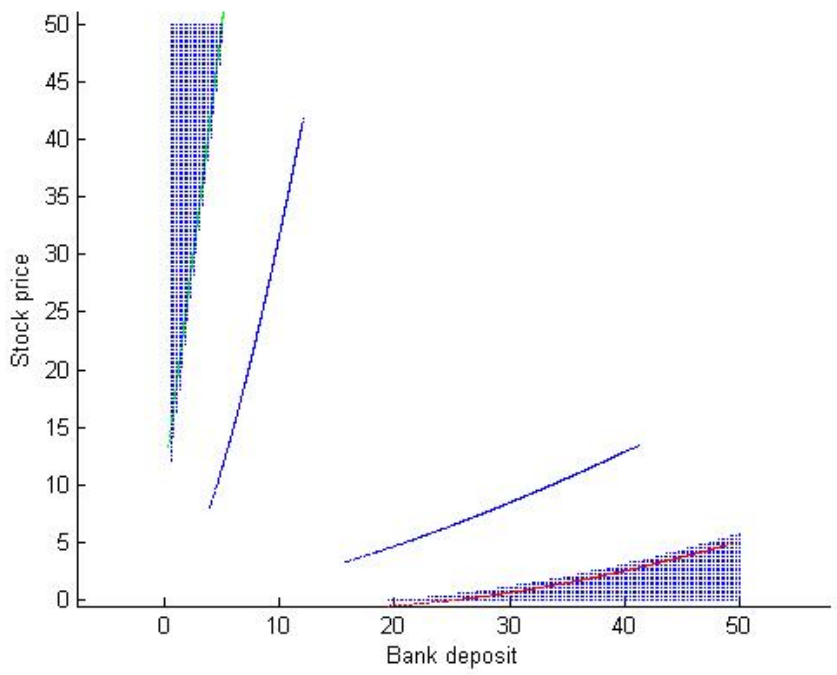

Figure 1.11: Transaction regions and NT region when $k=0.3, \lambda>0$ and $m=0.9318$.

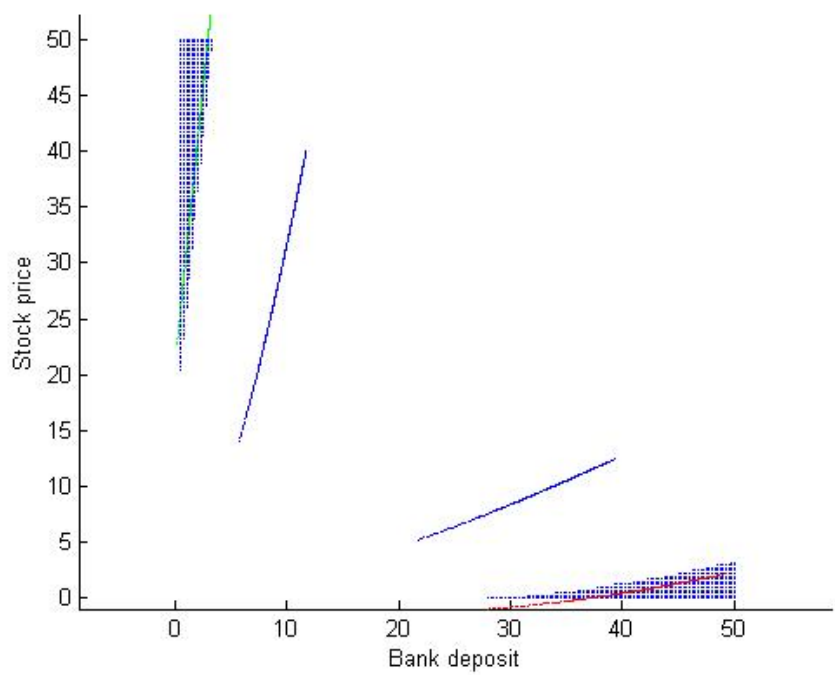

Figure 1.12: Transaction regions and NT region when $k=0.5, \lambda>0$ and $m=0.9318$. 
We assume that the value function is given by

$$
v(x, y)=a e^{b x}+\frac{y^{m}}{m}+\beta,
$$

where $a, b$, and $\beta$ are constants. Substituting (1.133) in (1.130) with $c^{*}$ given in (1.132) and collecting the like terms, we obtain

$$
\begin{gathered}
-r a b x e^{b x}+\frac{a b^{2}}{\gamma} x e^{b x}=0 \\
-\delta a e^{b x}+\frac{a b}{\gamma} \ln \left(\frac{a b}{\gamma}\right) e^{b x}-\frac{a b}{\gamma} e^{b x}=0, \\
-\delta \beta+1=0 .
\end{gathered}
$$

Therefore, $a=-\frac{1}{r} e^{\left(1-\frac{\delta}{r}\right)}, b=-r \gamma$ and $\beta=\frac{1}{\delta}$. Also, we may obtain $c^{*}=$ $r x-\frac{1}{\gamma}\left(1-\frac{\delta}{r}\right)$. Since the value of $m$ is exactly the same as in Theorem 1.1, we obtain (1.131).

Figure 1.13 shows the transaction regions and NT region for the exponential utility function (1.129).

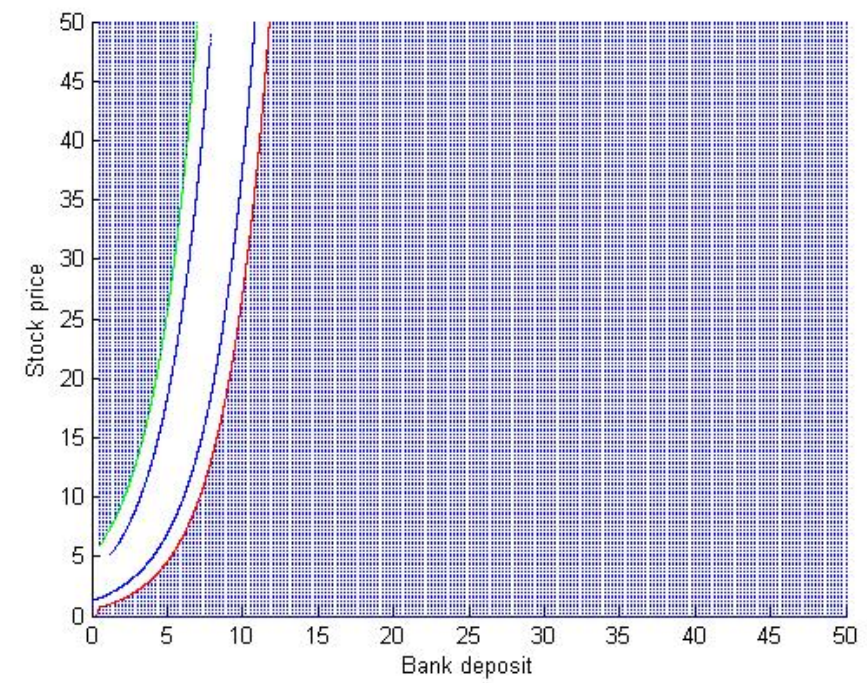

Figure 1.13: Transaction regions and NT region when $u(c)=1-e^{-\gamma c}, k>$ $0, \lambda>0$ and $m=0.9342$.

\subsubsection{Natural Logarithm utility function}

Theorem 1.16. Suppose that the utility function is a natural logarithm function given by

$$
u(c)=\ln c,
$$


then an explicit solution for the value function which satisfies

$$
\max _{c}\left[-\delta v+(r x-c) \frac{\partial v}{\partial x}+\alpha y \frac{\partial v}{\partial y}+\frac{1}{2} \sigma^{2} y^{2} \frac{\partial^{2} v}{\partial y^{2}}+u(c)\right]=0
$$

is given by

$$
v(x, y)=\frac{1}{\delta} \ln x+\frac{y^{m}}{m}+\frac{1}{\delta}\left(\frac{r-\delta}{\delta}+\ln \delta\right) .
$$

Proof. Differentiating (1.138) with respect to $c$ and solving for $c$, we have

$$
c^{*}=\left(\frac{\partial v}{\partial x}\right)^{-1} .
$$

We assume that the value function is given by

$$
v(x, y)=a \ln x+\frac{y^{m}}{m}+b,
$$

where $a$ and $b$ are constants. Substituting (1.141) in (1.138) with $c^{*}$ given in (1.140) and collecting the like terms, we obtain

$$
\begin{gathered}
-\delta a \ln x+\ln x=0, \\
-\delta b+r x \frac{a}{x}-\frac{x}{a} \frac{a}{x}-\ln a=0 .
\end{gathered}
$$

Therefore, $a=\frac{1}{\delta}$ and $b=\frac{1}{\delta}\left(\frac{r-\delta}{\delta}+\ln \delta\right)$, and since the value of $m$ is exactly the same as in Theorem 1.1, we obtain (1.139).

Remark 1.17. Notice that $c^{*}=\delta x$ from equation (1.140). This indicates that we always have to consume a proportional amount of money from the bank account.

Figure 1.14 shows the transaction regions and NT region for the natural logarithm utility function (1.137). 


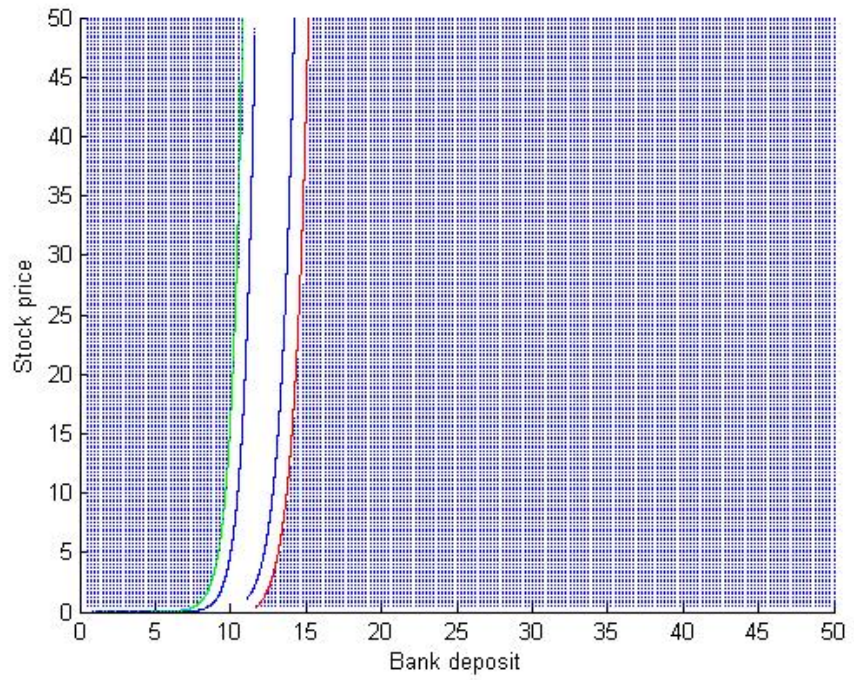

Figure 1.14: Transaction regions and NT region when $u(c)=\ln c, k>0, \lambda>0$ and $m=0.9342$. 


\section{Chapter 2}

\section{Multiple Risky Assets Problem}

\section{$2.1 \quad$ Introduction}

This chapter describes the optimal portfolio and consumption on multiple assets with transaction costs. We derive the explicit solutions to the HJB equations. We discuss the case with two risky assets and multiple risky assets. We maximize the time integral of the discounted utility function. Using the dynamic programing principle, we derive the Hamilton-Jacobi-Bellman (HJB) equation for the value function. Explicit solutions are found for the typical utility function which is a power function $u(c)=\frac{c^{\gamma}(t)}{\gamma}(0<\gamma<1)$, where $c(t)$ is the consumption rate.

Our contributions in this chapter are to distinguish and treat the money and the risky assets as multiple independent variables. The optimal consumption depends on the money but not on the risky assets. We find the explicit solutions to the HJB equations. Thanks to explicit solutions the intervention operator is reduced to a constrained optimization of the value functions. The theorem for the Viscosity solutions are proved in easier way also because of knowing the explicit solutions. Using the explicit solutions we are able to find the transaction and no-transaction regions, and the optimal portfolio curves for the two risky assets case. Also, we are able to discuss the algorithms for trading $N$ risky assets.

Merton [17] derive the optimality equations for a multiasset problem when the rate of returns are generated by a Wiener Brownian-motion process. Muthuraman and Kumar [19] provide a computational study of the problem of optimal allocating wealth among multiple stocks and a bank account, to maximize the infinite horizon discounted utility of consumption. Liu [15] shows that when asset returns are uncorrelated, the optimal investment policy is to keep the dollar amount invested in each risky asset between two constant levels and upon reaching either of these thresholds, to trade to the corresponding optimal targets. As 
the portfolio under consideration consists of multiple risky assets, which makes the numerical methods formidable, Atkinson and Ingpochai [4] apply perturbation analyses and also allow the risky assets to have correlation between their price processes. Inequalities for Hadamard product are given and proved in Jia [12].

The outline of this chapter is as follows. The mathematical formulation is given in section 2.2. In section 2.3, we discuss the transaction costs model with both proportional and fixed transaction costs, and obtain the explicit solution for the HJB equation. In section 2.4, we prove the explicit solutions for the HJB equation are viscosity solutions. Numerical results for two risky assets and $N$ risky assets as long as the algorithm for trading $N$ risky assets are discussed in section 2.5 .

\subsection{Formulation of the problem}

Let $(\Omega, F, P)$ be a probability space with $F(t)(t \geq 0)$ a given filtration. We define $S_{0}(t)(t \geq 0)$ as the amount of money invested in the money market (bank) and $S(t)=\left[S_{1}(t), S_{2}(t), \ldots, S_{N}(t)\right](t \geq 0)$ as the stochastic process of the amount of money invested in the $N$ stocks. We assume that the processes $S_{0}(t)$ and $S(t)$ satisfy

$$
\begin{gathered}
d S_{0}(t)=\left(r S_{0}(t)-c(t)\right) d t \quad \tau_{l} \leq t \leq \tau_{l+1}, \\
d S_{i}(t)=\alpha_{i} S_{i}(t) d t+S_{i}(t) \sum_{j=1}^{N} \sigma_{i j} d W_{j} \quad \tau_{l} \leq t \leq \tau_{l+1} \quad(i=1,2, \ldots, N), \\
S_{0}\left(\tau_{l+1}\right)=S_{0}\left(\tau_{l+1}^{-}\right)-k \widetilde{p}-\sum_{i=1}^{N} \xi_{i, l+1}-\lambda \sum_{i=1}^{N}\left|\xi_{i, l+1}\right| \quad(i=1,2, \ldots, N), \\
S_{i}\left(\tau_{l+1}\right)=S_{i}\left(\tau_{l+1}^{-}\right)+\xi_{i, l+1} \quad(i=1,2, \ldots, N), \\
S_{0}\left(0^{-}\right)=x \\
S_{i}\left(0^{-}\right)=y_{i},
\end{gathered}
$$

where $\xi_{i, l+1},(i=1,2, \ldots, N)$ are sizes of transactions for all $N$ stocks at time $\tau_{l+1}^{-}$and $\widetilde{p}$ is the number of how many $\xi_{i, l+1},(i=1,2, \ldots, N)$ which are not 0 . The control of the investor is a combination of a regular stochastic control $c(t)$ and an impulse control

$$
\phi=\left(\tau_{1}, \tau_{2}, \ldots ; \xi_{1,1}, \xi_{1,2}, \ldots, \xi_{1, N}, \xi_{2,1}, \xi_{2,2}, \ldots, \xi_{2, N}, \ldots, \xi_{N, 1}, \xi_{N, 2}, \ldots, \xi_{N, N}\right) .
$$

We consider the case where the shorts are not allowed. Therefore, we define a solvency region by

$$
S_{+}:=[0, \infty) \times[0, \infty) \times[0, \infty) \times \ldots \times[0, \infty) .
$$


We consider the problem using the utility function $u(c)=\frac{c^{\gamma}(t)}{\gamma}$ where $\gamma \in \Gamma:=$ $\{\gamma \in R: 0<\gamma<1\}$. The objective of the investor is to maximize the time integral of the discounted utility function for his life time consumption or to find the value function

$$
\widetilde{\Phi}(s, x, y)=\sup _{c, \phi} E\left[\int_{0}^{\infty} e^{-\delta(s+t)} \frac{c^{\gamma}(t)}{\gamma} d t\right],
$$

where $\delta>0$ is a constant discount rate and $y=\left(y_{1}, y_{2}, \ldots, y_{N}\right)$. We assume that $\alpha>\delta$. For the case $\alpha<\delta$, in particular $\alpha<0$, this means stocks will go down and it is better to sell the stocks. Note that $1-\gamma$ is called the relative risk aversion coefficient. In the infinite horizon problem we can simplify the problem in the following manner

$$
\widetilde{\Phi}(s, x, y)=e^{-\delta s} \sup _{c, \phi} E\left[\int_{0}^{\infty} e^{-\delta t} \frac{c^{\gamma}(t)}{\gamma} d t\right]=e^{-\delta s} \widetilde{\Phi}(0, x, y) \triangleq e^{-\delta s} \Phi(x, y),
$$

so that we seek a time independent value function $\Phi(x, y)$.

Similarly as we did for the one risky asset, we need to distinguish the case where there are no transactions and the case where the transactions are taking place. First we discuss the no-transaction case. Let $\tilde{v}(s, x, y)=e^{-\delta s} v(x, y)$ be the value function for the no-transaction case and using the dynamical principle, we see that $v$ satisfies the Hamilton-Jacobi-Bellman (HJB) equation given by

$$
\max _{c}\left[-\delta v+(r x-c) \frac{\partial v}{\partial x}+\sum_{i=1}^{N} \alpha_{i} y_{i} \frac{\partial v}{\partial y_{i}}+\frac{1}{2} \sum_{i, j=1}^{N} \sigma_{i j} y_{i} y_{j} \frac{\partial^{2} v}{\partial y_{i} \partial y_{j}}+\frac{c^{\gamma}}{\gamma}\right]=0 .
$$

We define

$$
L^{c} v(x, y)=-\delta v+(r x-c) \frac{\partial v}{\partial x}+\sum_{i=1}^{N} \alpha_{i} y_{i} \frac{\partial v}{\partial y_{i}}+\frac{1}{2} \sum_{i, i=1}^{N} \sigma_{i j} y_{i} y_{j} \frac{\partial^{2} v}{\partial y_{i} \partial y_{j}} .
$$

Also, we define

$$
\begin{gathered}
L v(x, y)=\sup _{c \geq 0}\left\{L^{c} v(x, y)+\frac{c^{\gamma}}{\gamma}\right\}, \quad(x, y) \in S_{+}, \\
L_{0} v(0, y)=-\delta v+\sum_{i=1}^{N} \alpha_{i} y_{i} \frac{\partial v}{\partial y_{i}}+\frac{1}{2} \sum_{i, i=1}^{N} \sigma_{i j} y_{i} y_{j} \frac{\partial^{2} v}{\partial y_{i} \partial y_{j}} .
\end{gathered}
$$

On the boundaries where $y_{i}(i=1,2, \ldots, N)$ could be 0 , the situation where several $y_{i}$ s are zero and the rest of $y_{i}$ s are non-zero may take place.

Let $v: S_{+} \rightarrow R^{+}$be a measurable function. The intervention operator $M$ is defined by

$$
M v(x, y)=\sup _{\xi}\left\{v\left(x^{\prime}, y^{\prime}\right): \xi \in R \backslash\{0\},\left(x^{\prime}, y^{\prime}\right) \in S_{+}\right\},
$$


where

$$
x^{\prime}(\xi)=x-k \widetilde{p}-\sum_{i=1}^{N} \xi_{i}-\lambda \sum_{i=1}^{N}\left|\xi_{i}\right|,
$$

here $\widetilde{p}$ is the number of nonzero transactions, and

$$
y^{\prime}(\xi)=y+\xi .
$$

Here, $k$ and $\lambda$ are a fixed transaction cost and a proportional transaction cost of trading respectively. We compare the current value $v$ and the optimal value $M v$ after the trading. The region where $M v \geq v$ is called the transaction region and the region where $M v<v$ is called the no-transaction region and denoted by $N T$. We also call the set of $(x, y) \in S_{+}$at which $M v(x, y)=v(x, y)$ the transaction boundaries.

\subsection{Solutions to HJB equation}

We consider the equation (2.11) first. Concerning the solutions of (2.11), we have

Theorem 2.1. A solution to (2.11) is given by

$$
\begin{aligned}
& v\left(x, y_{1}, y_{2}, \ldots, y_{N}\right)=a x^{\gamma}+\sum_{i=1}^{N} b_{i} y_{i}^{m_{i}}+\sum_{i_{1}, i_{2}=1, i_{1} \neq i_{2}}^{N} b_{i_{1} i_{2}}\left(y_{i_{1}} y_{i_{2}}\right)^{m_{i_{1} i_{2}}} \\
&+\sum_{i_{1}, i_{2}, i_{3}=1, i_{1} \neq i_{2} \neq i_{3}}^{N} b_{i_{1} i_{2} i_{3}}\left(y_{i_{1}} y_{i_{2}} y_{i_{3}}\right)^{m_{i_{1} i_{2} i_{3}}} \\
&+\sum_{i_{1}, i_{2}, \ldots, i_{k}=1, i_{1} \neq i_{2} \neq \ldots \neq i_{k}} b_{i_{1} i_{2} \ldots i_{k}}\left(y_{i_{1}} y_{i_{2} \ldots} y_{i_{k}}\right)^{m_{i_{1} i_{2} \ldots i_{k}}} \\
&+\ldots+\sum_{i_{1}, i_{2}, \ldots, i_{N}=1, i_{1} \neq i_{2} \neq \ldots \neq i_{N}}^{N} b_{i_{1} i_{2} \ldots i_{N}}\left(y_{i_{1}} y_{i_{2} \ldots} y_{i_{N}}\right)^{m_{i_{1} i_{2} \ldots i_{N}}},
\end{aligned}
$$

where

$$
a=\frac{1}{\gamma}\left[\frac{1}{1-\gamma}(\delta-r \gamma)\right]^{\gamma-1}
$$

and

$$
\begin{aligned}
= & \frac{m_{i_{1} i_{2} \ldots i_{k}}}{\left(\frac{1}{2} \sum_{i=1}^{k} \sigma_{i i}-\sum_{i=1}^{k} \alpha_{i}\right) \pm \sqrt{\left(\sum_{i=1}^{k} \alpha_{i}-\frac{1}{2} \sum_{i=1}^{k} \sigma_{i i}\right)^{2}+2 \delta \sum_{p, q=1}^{k} \sigma_{i_{p} i_{q}}}} \\
& (k=1,2, \ldots, N) .
\end{aligned}
$$

The $b_{i_{1} i_{2} \ldots i_{k}}(k=1,2, \ldots, N)$ in (2.18) are positive real numbers. 
Proof. Differentiating (2.11) with respect to $c$, we have $c^{*}=\left(\frac{\partial v}{\partial x}\right)^{\frac{1}{\gamma-1}}$. Substituting $c^{*}$ into (2.11), we obtain

$$
-\delta v+r x \frac{\partial v}{\partial x}+\sum_{i=1}^{N} \alpha_{i} y_{i} \frac{\partial v}{\partial y_{i}}+\frac{1}{2} \sum_{i, j=1}^{N} \sigma_{i j} y_{i} y_{j} \frac{\partial^{2} v}{\partial y_{i} \partial y_{j}}+\frac{1-\gamma}{\gamma}\left(\frac{\partial v}{\partial x}\right)^{\frac{\gamma}{\gamma-1}}=0 .
$$

Substituting the ansatz (2.18) into (2.21) and collecting the like terms, we get two equations

$$
\left[-\delta a+r a \gamma+\frac{1-\gamma}{\gamma} a^{\frac{\gamma}{\gamma-1}} \gamma^{\frac{\gamma}{\gamma-1}}\right] x^{\gamma}=0
$$

and

$$
-\delta v+\sum_{i=1}^{N} \alpha_{i} y_{i} \frac{\partial v}{\partial y_{i}}+\frac{1}{2} \sum_{i, j=1}^{N} \sigma_{i j} y_{i} y_{j} \frac{\partial^{2} v}{\partial y_{i} \partial y_{j}}=0 .
$$

Since each $y$ terms in equation (2.18) satisfies (2.23), from equations (2.22) and (2.23) we get (2.19) and (2.20).

Remark 2.2. A more general form for the explicit solution to (2.11) is: the $y$ terms in equation (2.18) have the forms of $y_{i_{1}}^{m_{i_{1}}} y_{i_{2}}^{m_{i_{2}} \ldots y_{i_{k}}^{m_{i_{k}}}}\left(m_{i_{1}} \geq 0, m_{i_{2}} \geq\right.$ $\left.0, \ldots, m_{i_{k}} \geq 0\right)$.

Since we know an explicit solution in the no transaction region, the problem is reduced to finding $\max [v(x, y), M v(x, y)]$ for each $(x, y) \in S_{+}$. Combining the no-transaction and transaction cases, we define

$$
\Phi(x, y)=\max [v(x, y), M v(x, y)]
$$

as the value function. Therefore, we obtain a quasi-variational inequality problem

$$
\max _{c, \phi}\left\{L^{c} v+\frac{c^{\gamma}}{\gamma}, M v-\Phi(x, y)\right\}=0
$$

with the understanding that if both $L^{c} v+\frac{1}{\gamma} c^{\gamma}$ and $M v-\Phi(x, y)$ are equal to zero, we make trades and move to a new allocation. With this formulation we can handle both fixed and proportional transaction costs.

Lemma 2.3. (Concavity of the value function) A simplified explicit solution satisfies (2.11) is given by

$$
v\left(x, y_{1}, y_{2}, \ldots, y_{N}\right)=a x^{\gamma}+\sum_{i=1}^{N} b_{i} y_{i}^{m_{i}}+\sum_{i, j=1, i \neq j}^{N} b_{i j}\left(y_{i} y_{j}\right)^{m_{i j}} .
$$

The value function given by (2.26) is concave if $0<m_{i}<1,0<m_{i j}<0.5$ for $i, j=1,2, \ldots N,(i \neq j)$.

Proof. When $0<\gamma<1$ and $0<m_{i}<1, f(x)=x^{\gamma}$ and $g_{i}\left(y_{i}\right)=y_{i}^{m_{i}}$ are all concave functions for $i=1,2, \ldots, N$. In order to prove the value function given by (2.26) is a concave function, next we show when $0<m_{i j}<0.5$, 
$h_{i j}\left(y_{i}, y_{j}\right)=b_{i j}\left(y_{i} y_{j}\right)^{m_{i j}}$ are concave functions for $i, j=1,2, \ldots N,(i \neq j)$. The Hessian of $-\sum_{i, j=1, i \neq j}^{N} b_{i j}\left(y_{i} y_{j}\right)^{m_{i j}}$ is given below,

$$
\begin{aligned}
& H\left(y_{i}, y_{j}\right) \\
& \left.=\left(\begin{array}{cc}
-b_{i j} m_{i j}\left(m_{i j}-1\right) y_{i}^{m_{i j}-2} y_{j}^{m_{i j}} & -b_{i j} m_{i j}^{2} y_{i}^{m_{i j}-1} y_{j}^{m_{i j}-1} \\
-b_{i j} m_{i j}^{2} y_{i}^{m_{i j}-1} y_{j}^{m_{i j}-1} & -b_{i j} m_{i j}\left(m_{i j}-1\right) y_{i}^{m_{i j}} y_{j}^{m_{i j}-2}
\end{array}\right) 2.27\right)
\end{aligned}
$$

The determinant of $H$ is calculated, and

$$
\begin{aligned}
& \left|H\left(y_{i}, y_{j}\right)\right| \\
= & \left|\begin{array}{cc}
-b_{i j} m_{i j}\left(m_{i j}-1\right) y_{i}^{m_{i j}-2} \cdot y_{j}^{m_{i j}} & -b_{i j} m_{i j}^{2} y_{i}^{m_{i j}-1} \cdot y_{j}^{m_{i j}-1} \\
-b_{i j} m_{i j}^{2} y_{i}^{m_{i j}-1} \cdot y_{j}^{m_{i j}-1} & -b_{i j} m_{i j}\left(m_{i j}-1\right) y_{i}^{m_{i j}} \cdot y_{j}^{m_{i j}-2}
\end{array}\right| \\
= & \left(b_{i j} m_{i j}\right)^{2} y_{i}^{2 m_{i j}-2} \cdot y_{j}^{2 m_{i j}-2} \cdot\left|\begin{array}{cc}
m_{i j}-1 & m_{i j} \\
m_{i j} & m_{i j}-1
\end{array}\right| \\
= & \left(b_{i j} m_{i j}\right)^{2} y_{i}^{2 m_{i j}-2} \cdot y_{j}^{2 m_{i j}-2} \cdot\left(1-2 m_{i j}\right)>0
\end{aligned}
$$

if $m_{i j}<0.5,(i \neq j)$. Thus, the value function given by (2.26) is concave if $0<m_{i}<1,0<m_{i j}<0.5$ for $i, j=1,2, \ldots N,(i \neq j)$.

\subsection{Viscosity Solution}

Since we know that (2.18) in Theorem 2.1 is an explicit solution to the HJB equation and we define the value function $\Phi$ which is given in (2.24), it is interesting to prove that $\Phi$ is a viscosity solution for the quasi-variational inequality problem given in (2.25).

Definition 2.4. Suppose $G$ is a topological space, $x_{0}$ is a point in $G$ and $\psi: G \rightarrow R$ is a function.

(1)We say that $\varphi$ is upper semi-continuous (usc) at $x_{0}$ if $\limsup _{x \rightarrow x_{0}} \varphi(x) \leq \varphi\left(x_{0}\right)$. The function $\varphi$ is called upper semi-continuous if it is upper semi-continuous at every point of $G$.

(2) Similarly, we say that $\varphi$ is lower semi-continuous (lsc) at $x_{0}$ if $\liminf _{x \rightarrow x_{0}} \varphi(x) \geq$ $\varphi\left(x_{0}\right)$. The function $\varphi$ is called lower semi-continuous if it is lower semicontinuous at every point of $G$.

(3) We let $U S C(G)$ and $L S C(G)$ denote the set of usc functions and $l s c$ functions on $G$, respectively.

Definition 2.5. Let $S_{+}^{0}$ be the interior of $S_{+}$and $\zeta=\left(x, y_{1}, y_{2}, \ldots, y_{N}\right)$. We 
define $F$ as follows.

$$
\begin{aligned}
& F\left(D^{2} v(\zeta), D v(\zeta), v(\zeta), \zeta\right) \\
& =\left\{\begin{array}{l}
\max _{c, \phi}\left\{-\delta v+(r x-c) \frac{\partial v}{\partial x}+\sum_{i=1}^{N} \alpha_{i} y_{i} \frac{\partial v}{\partial y_{i}}+\frac{1}{2} \sum_{i, j=1}^{N} \sigma_{i j} y_{i} y_{j} \frac{\partial^{2} v}{\partial y_{i} \partial y_{j}}+\frac{c^{\gamma}}{\gamma},\right. \\
M v-\Phi\}, \text { if } \zeta \in S_{+}^{0} ; \\
\max _{c, \phi}\left\{-\delta v+(r x-c) \frac{\partial v}{\partial x}+\sum_{j=1, j \neq i_{1}, \ldots, j \neq i_{k}}^{N} \alpha_{j} y_{j} \frac{\partial v}{\partial y_{j}}\right. \\
\left.+\frac{1}{2} \sum^{N} j=1, j \neq i_{1}, \ldots, j \neq i_{k} \quad \sigma_{j l} y_{j} y_{l} \frac{\partial^{2} v}{\partial y_{j} \partial y_{l}}+\frac{c^{\gamma}}{\gamma}, M v-\Phi\right\} \\
\quad l=1, l \neq i_{1}, \ldots, l \neq i_{k} \\
\text { if } y_{i_{1}}=0 \& y_{i_{2}}=0 \& \ldots \& y_{i_{k}}=0, \text { and } x \in(0, \infty) ; \\
\max _{\phi}\left\{-\delta v+\sum_{j=1, j \neq i_{1}, \ldots, j \neq i_{k}}^{N} \alpha_{j} y_{j} \frac{\partial v}{\partial y_{j}}\right. \\
+\frac{1}{2} \sum^{N} j=1, j \neq i_{1}, \ldots, j \neq i_{k} \\
\left.\quad \sigma_{j l} y_{j} y_{l} \frac{\partial^{2} v}{\partial y_{j} \partial y_{l}}, M v-\Phi\right\} \\
\text { if } x=0 \& y_{i_{1}}=0 \& y_{i_{2}}=0 \& \ldots \& y_{i_{k}}=0 \\
\text { and }\left(y_{i_{k+1}}, y_{i_{k+2}}, \ldots, y_{N}\right) \in(0, \infty) \times(0, \infty) \times \ldots \times(0, \infty) .
\end{array}\right.
\end{aligned}
$$

(1) A function $\psi \in U S C\left(S_{+}\right)$is a viscosity subsolution of

$$
F\left(D^{2} v(\zeta), D v(\zeta), v(\zeta), \zeta\right)=0
$$

if for every $\zeta_{0}=\left(x_{0}, y_{01}, y_{02}, \ldots, y_{0 N}\right) \in S_{+}$and for every function $f \in C^{2}\left(S_{+}\right)$ satisfying $f \geq \psi$ on $S_{+}$and $f\left(\zeta_{0}\right)=\psi\left(\zeta_{0}\right)$, we have

$$
F\left(D^{2} f\left(\zeta_{0}\right), D f\left(\zeta_{0}\right), f\left(\zeta_{0}\right), \zeta_{0}\right) \geq 0 .
$$

(2) A function $\psi \in L S C\left(S_{+}\right)$is a viscosity supersolution of (2.30) if for every $\zeta_{0} \in S_{+}$and for every function $f \in C^{2}\left(S_{+}\right)$satisfying $f \leq \psi$ on $S_{+}$and $f\left(\zeta_{0}\right)=\psi\left(\zeta_{0}\right)$, we have

$$
F\left(D^{2} f\left(\zeta_{0}\right), D f\left(\zeta_{0}\right), f\left(\zeta_{0}\right), \zeta_{0}\right) \leq 0 .
$$

(3) A function $\psi: S_{+} \rightarrow R$ is a viscosity solution of (2.30) if $\psi$ is locally bounded and it is a viscosity sub and super solution of (2.30).

Lemma 2.6. The variance and covariance matrix

$$
\sum=\left(\sigma_{i j}\right)=\operatorname{Cov}\left[S_{i}, S_{j}\right] i, j=1,2, \ldots N,
$$

of the $N$ risky assets $S=\left(S_{1}, S_{2}, \ldots, S_{N}\right)$, is positive semidefinite. 
Proof. For any vector $z \in R^{n}$, and the symmetry of $\sum$, we obtain

$$
\begin{aligned}
z^{T} \sum z & =\sum_{i=1}^{N} \sum_{j=1}^{N}\left(\sigma_{i j} z_{i} z_{j}\right) \\
& =\sum_{i=1}^{N} \sum_{j=1}^{N}\left(\operatorname{Cov}\left[S_{i}, S_{j}\right] z_{i} z_{j}\right) \\
& =\sum_{i=1}^{N} \sum_{j=1}^{N}\left(E\left[\left(S_{i}-E\left[S_{i}\right]\right)\left(S_{j}-E\left[S_{j}\right]\right)\right] z_{i} z_{j}\right) \\
& =E\left[\sum_{i=1}^{N} \sum_{j=1}^{N}\left(S_{i}-E\left[S_{i}\right]\right)\left(S_{j}-E\left[S_{j}\right]\right) z_{i} z_{j}\right] \\
& =E\left[(S-E[S])^{T} z\right]^{2} \geq 0 .
\end{aligned}
$$

Lemma 2.7. The Hadamard product matrix

$$
A \star B=\left(a_{i j}\right)_{N \times N} \star\left(b_{i j}\right)_{N \times N}=\left(a_{i j} \star b_{i j}\right)_{N \times N},
$$

is positive semidefinite, if both of matrices $A=\left(a_{i j}\right)_{N \times N}$ and $B=\left(b_{i j}\right)_{N \times N}$ are positive semidefinite.

Proof. Let $\operatorname{rank}(B)=\widetilde{r}$, so there exists a matrix $G_{r \times N}$ such that $B=G^{T} G$. If we denote $G^{T}=\left(g_{i j}\right)_{N \times r}$, then $b_{i j}=\sum_{l=1}^{\widetilde{r}} g_{i l} g_{j l}$. For any vector $z \in R^{n}$,

$$
\begin{aligned}
z^{T}(A \star B) z & =\sum_{i=1}^{N} \sum_{j=1}^{N} z_{i} a_{i j} b_{i j} z_{j} \\
& =\sum_{i=1}^{N} \sum_{j=1}^{N} z_{i} a_{i j}\left(\sum_{l=1}^{\widetilde{r}} g_{i l} g_{j l}\right) z_{j} \\
& =\sum_{l=1}^{\widetilde{r}}\left[\sum_{i=1}^{N} \sum_{j=1}^{N} a_{i j}\left(z_{i} g_{i l}\right)\left(z_{j} g_{j l}\right)\right] \geq 0,
\end{aligned}
$$

since matrix $A$ is positive semidefinite [12].

Theorem 2.8. The value function $\Phi$ is a viscosity solution of (2.30).

Proof. (1) For $\zeta=\left(x, y_{1}, y_{2}, \ldots, y_{N}\right) \in S_{+}^{0}$, we prove that $\Phi(\zeta)=\max [v(\zeta), M v(\zeta)]$ is a viscosity subsolution of the first case in (2.29). It is easy to see that $v$ is $C^{2}$. Consider that $f$ is a $C^{2}$ function in a neighborhood of $S_{+}^{0}$ and let $\zeta_{0}=\left(x_{0}, y_{01}, y_{02}, \ldots, y_{0 N}\right) \in S_{+}^{0}$ be such that $f \geq \Phi$ on $S_{+}^{0}$ and $f\left(\zeta_{0}\right)=\Phi\left(\zeta_{0}\right)$. We show two cases separately. 
Case (i). $v\left(\zeta_{0}\right) \leq M v\left(\zeta_{0}\right)$. Then, $f\left(\zeta_{0}\right)=\Phi\left(\zeta_{0}\right)=\max \left[v\left(\zeta_{0}\right), M v\left(\zeta_{0}\right)\right]=$ $M v\left(\zeta_{0}\right)$. Since

$$
M v\left(\zeta_{0}\right)=v\left(x^{\prime}, y_{1}^{\prime}, y_{2}^{\prime}, \ldots, y_{N}^{\prime}\right) \leq f\left(x^{\prime}, y_{1}^{\prime}, y_{2}^{\prime}, \ldots, y_{N}^{\prime}\right) \leq M f\left(\zeta_{0}\right),
$$

we have

$$
\begin{aligned}
& \max _{c, \phi}\left\{\left[-\delta f+(r x-c) \frac{\partial f}{\partial x}+\sum_{i=1}^{N} \alpha_{i} y_{i} \frac{\partial f}{\partial y_{i}}+\frac{1}{2} \sum_{i, j=1}^{N} \sigma_{i j} y_{i} y_{j} \frac{\partial^{2} f}{\partial y_{i} \partial y_{j}}+\frac{c^{\gamma}}{\gamma}\right]\left(\zeta_{0}\right),\right. \\
& {\left.[M f-\max (M f, f)]\left(\zeta_{0}\right)\right\} } \\
\geq & {[M f-\max (M f, f)]\left(\zeta_{0}\right) } \\
= & {[M f-\max (M f, M v)]\left(\zeta_{0}\right) \geq 0 . }
\end{aligned}
$$

So (2.31) holds.

Case (ii). $v\left(\zeta_{0}\right)>M v\left(\zeta_{0}\right)$. We need to prove

$$
\begin{aligned}
& \max _{c}\left[-\delta f+(r x-c) \frac{\partial f}{\partial x}+\sum_{i=1}^{N} \alpha_{i} y_{i} \frac{\partial f}{\partial y_{i}}+\frac{1}{2} \sum_{i, j=1}^{N} \sigma_{i j} y_{i} y_{j} \frac{\partial^{2} f}{\partial y_{i} \partial y_{j}}+\frac{c^{\gamma}}{\gamma}\right]\left(\zeta_{0}\right) \\
\geq & 0 .
\end{aligned}
$$

Since $f \geq \Phi \geq v$ on $S_{+}^{0}$ and $f\left(\zeta_{0}\right)=\Phi\left(\zeta_{0}\right)=v\left(\zeta_{0}\right), f-v$ has a local minimum at $\zeta_{0}$. Therefore, $\frac{\partial f}{\partial x}\left(\zeta_{0}\right)=\frac{\partial v}{\partial x}\left(\zeta_{0}\right), \frac{\partial f}{\partial y_{i}}\left(\zeta_{0}\right)=\frac{\partial v}{\partial y_{i}}\left(\zeta_{0}\right),(i=1,2, \ldots, N)$ and the matrix $\left[\frac{\partial^{2}(f-v)}{\partial y_{i} \partial y_{j}}\right]_{N \times N}$ is positive semidefinite [7]. By using Lemma 2.7, $\sum_{i, j=1}^{N} \sigma_{i j} y_{i} y_{j} \frac{\partial^{2} f}{\partial y_{i} \partial y_{j}} \geq \sum_{i, j=1}^{N} \sigma_{i j} y_{i} y_{j} \frac{\partial^{2} v}{\partial y_{i} \partial y_{j}}$ becasue both of the matrices $\left(\sigma_{i j}\right)_{N \times N}$ and $\left[\frac{\partial^{2}(f-v)}{\partial y_{i} \partial y_{j}}\right]_{N \times N}$ are positive semidefinite. This implies

$$
\begin{aligned}
\max _{c}\left[-\delta f+(r x-c) \frac{\partial f}{\partial x}+\sum_{i=1}^{N} \alpha_{i} y_{i} \frac{\partial f}{\partial y_{i}}\right. \\
\left.+\frac{1}{2} \sum_{i, j=1}^{N} \sigma_{i j} y_{i} y_{j} \frac{\partial^{2} f}{\partial y_{i} \partial y_{j}}+\frac{c^{\gamma}}{\gamma}\right]\left(\zeta_{0}\right) \\
\geq \max _{c}\left[-\delta v+(r x-c) \frac{\partial v}{\partial x}+\sum_{i=1}^{N} \alpha_{i} y_{i} \frac{\partial v}{\partial y_{i}}\right. \\
\left.+\frac{1}{2} \sum_{i, j=1}^{N} \sigma_{i j} y_{i} y_{j} \frac{\partial^{2} v}{\partial y_{i} \partial y_{j}}+\frac{c^{\gamma}}{\gamma}\right]\left(\zeta_{0}\right) .
\end{aligned}
$$

We choose $c^{*}=\left(\frac{\partial v}{\partial x}\right)^{\frac{1}{\gamma-1}}$ and substitute the value function (2.18) into the right hand side of (2.39). By Theorem 2.1, the right hand side of (2.39) is zero.

The proofs for the cases where $x=0 \& y_{i_{1}}=0 \& y_{i_{2}}=0 \& \ldots \& y_{i_{k}}=0$ or $y_{i_{1}}=$ $0 \& y_{i_{2}}=0 \& \ldots \& y_{i_{k}}=0$ are similar. 
(2) For $\zeta \in S_{+}^{0}$, we prove that $\Phi(\zeta)=\max [v(\zeta), M v(\zeta)]$ is a viscosity supersolution of the first case in (2.29). So we let $f$ be a $C^{2}$ function in a neighbourhood of $S_{+}^{0}$ and we let $\zeta_{0} \in S_{+}^{0}$ such that $f \leq \Phi$ on $S_{+}^{0}$ and $f\left(\zeta_{0}\right)=\Phi\left(\zeta_{0}\right)$. We want to show (2.32).

Since $[M f-\max (M f, f)]\left(\zeta_{0}\right) \leq 0$, we only need to prove

$$
\max _{c}\left[-\delta f+(r x-c) \frac{\partial f}{\partial x}+\sum_{i=1}^{N} \alpha_{i} y_{i} \frac{\partial f}{\partial y_{i}}+\frac{1}{2} \sum_{i, j=1}^{N} \sigma_{i j} y_{i} y_{j} \frac{\partial^{2} f}{\partial y_{i} \partial y_{j}}+\frac{c^{\gamma}}{\gamma}\right]\left(\zeta_{0}\right) \leq 0 .
$$

For $\bar{\varepsilon}>0$ and $\bar{w}=\bar{w}_{\bar{\varepsilon}, c}$ be an admissible control begining with a constant consumption rate $c>0$ and no transactions up to the first time $\bar{\tau}_{\bar{\varepsilon}}$ at which the stochastic process exists from

$$
\begin{aligned}
& \bar{K}_{\bar{\varepsilon}} \\
= & \left\{\left(s, x, y_{1}, y_{2}, \ldots, y_{N}\right) ;\left|\left(s, x, y_{1}, y_{2}, \ldots, y_{N}\right)-\left(0, x_{0}, y_{01}, y_{02}, \ldots, y_{0 N}\right)\right|<\bar{\varepsilon}\right\} \\
& \cap \bar{S}_{+},
\end{aligned}
$$

where $\zeta_{0}=\left(x_{0}, y_{01}, y_{02}, \ldots, y_{0 N}\right)$ and $\bar{S}_{+}=R^{+} \times S_{+}$. Choose $\zeta_{n} \in \bar{K}_{\bar{\varepsilon}}$ such that $\zeta_{n} \rightarrow \zeta_{0}$ and $\Phi\left(\zeta_{n}\right) \rightarrow \Phi\left(\zeta_{0}\right)$ as $n \rightarrow \infty$. By using It $\widehat{o}$-Doeblin formula and the definition of expectation, we obtain

$$
\begin{aligned}
& \Phi\left(\zeta_{n}\right) \\
\geq & E^{0, \zeta_{n}}\left[e^{-\delta \bar{\tau}_{\bar{\varepsilon}}} \Phi\left(X^{\bar{w}}\left(\bar{\tau}_{\bar{\varepsilon}}\right), Y_{1}^{\bar{w}}\left(\bar{\tau}_{\bar{\varepsilon}}\right), \ldots, Y_{N}^{\bar{w}}\left(\bar{\tau}_{\bar{\varepsilon}}\right)\right)\right]+E^{0, \zeta_{n}}\left[\int_{0}^{\bar{\tau}_{\bar{\varepsilon}}} e^{-\delta t} \frac{c^{\gamma}(X(t))}{\gamma} d t\right] \\
\geq & E^{0, \zeta_{n}}\left[e^{-\delta \bar{\tau}_{\bar{\varepsilon}}} f\left(X^{\bar{w}}\left(\bar{\tau}_{\bar{\varepsilon}}\right), Y_{1}^{\bar{w}}\left(\bar{\tau}_{\bar{\varepsilon}}\right), \ldots, Y_{N}^{\bar{w}}\left(\bar{\tau}_{\bar{\varepsilon}}\right)\right)\right]+E^{0, \zeta_{n}}\left[\int_{0}^{\bar{\tau}_{\bar{\varepsilon}}} e^{-\delta t} \frac{c^{\gamma}(X(t))}{\gamma} d t\right] \\
= & f\left(\zeta_{n}\right)+E^{0, \zeta_{n}}\left[\int_{0}^{\bar{\tau}_{\bar{\varepsilon}}} e^{-\delta t} L^{c} f\left(X^{\bar{w}}(t), Y_{1}^{\bar{w}}(t), \ldots, Y_{N}^{\bar{w}}(t)\right) d t\right] \\
& +E^{0, \zeta_{n}}\left[\int_{0}^{\bar{\tau}_{\bar{\varepsilon}}} e^{-\delta t} \frac{c^{\gamma}(X(t))}{\gamma} d t\right],
\end{aligned}
$$

where

$$
\begin{aligned}
& L^{c} f\left(x, y_{1}, y_{2}, \ldots, y_{N}\right) \\
= & -\delta f+(r x-c) \frac{\partial f}{\partial x}+\sum_{i=1}^{N} \alpha_{i} y_{i} \frac{\partial f}{\partial y_{i}}+\frac{1}{2} \sum_{i, j=1}^{N} \sigma_{i j} y_{i} y_{j} \frac{\partial^{2} f}{\partial y_{i} \partial y_{j}}
\end{aligned}
$$

and for all $n$. From (2.42),

$$
\begin{aligned}
& E^{0, \zeta_{n}}\left[\int_{0}^{\bar{\tau}_{\bar{\varepsilon}}} e^{-\delta t}\left\{L^{c} f\left(X^{\bar{w}}(t), Y_{1}^{\bar{w}}(t), \ldots, Y_{N}^{\bar{w}}(t)\right)+\frac{c^{\gamma}(X(t))}{\gamma}\right\} d t\right] \\
\leq & \Phi\left(\zeta_{n}\right)-f\left(\zeta_{n}\right)
\end{aligned}
$$

for all $n$. Taking the limit as $n \rightarrow \infty$, we obtain

$$
E^{0, \zeta_{0}}\left[\int_{0}^{\bar{\tau}_{\bar{\varepsilon}}} H(t) d t\right] \leq 0
$$


where

$$
H(t)=e^{-\delta t}\left(L^{c} f\left(X^{\bar{w}}(t), Y_{1}^{\bar{w}}(t), \ldots, Y_{N}^{\bar{w}}(t)\right)+\frac{c^{\gamma}(X(t))}{\gamma}\right) .
$$

By dividing the left-hand side by $E^{0, \zeta_{0}}\left[\bar{\tau}_{\bar{\varepsilon}}\right]$ we get

$$
\frac{E^{0, \zeta_{0}}\left[\int_{0}^{\bar{\tau}_{\bar{\varepsilon}}} H(t) d t\right]}{E^{0, \zeta_{0}}\left[\bar{\tau}_{\bar{\varepsilon}}\right]}=\frac{E^{0, \zeta_{0}}\left[\int_{0}^{\bar{\tau}_{\bar{\varepsilon}}}[H(t)-H(0)] d t\right]+H(0) E^{0, \zeta_{0}}\left[\bar{\tau}_{\bar{\varepsilon}}\right]}{E^{0, \zeta_{0}}\left[\bar{\tau}_{\bar{\varepsilon}}\right]} \rightarrow H(0)
$$

as $\bar{\varepsilon} \rightarrow 0$, since $H(t)$ is continous at $t=0$. Thus, we have

$H(0)=\left[-\delta f+(r x-c) \frac{\partial f}{\partial x}+\sum_{i=1}^{N} \alpha_{i} y_{i} \frac{\partial f}{\partial y_{i}}+\frac{1}{2} \sum_{i, j=1}^{N} \sigma_{i j} y_{i} y_{j} \frac{\partial^{2} f}{\partial y_{i} \partial y_{j}}+\frac{c^{\gamma}}{\gamma}\right]\left(\zeta_{0}\right) \leq 0$

for all $c \geq 0$ such that $\bar{w}_{\bar{\varepsilon}, c}$ is admissible for $\bar{\varepsilon}$ small enough [20].

The proofs for the cases where $x=0 \& y_{i_{1}}=0 \& y_{i_{2}}=0 \& \ldots \& y_{i_{k}}=0$ or $y_{i_{1}}=$ $0 \& y_{i_{2}}=0 \& \ldots \& y_{i_{k}}=0$ are similar.

(3) (1) and (2) imply that $\Phi$ is a viscosity solution of (2.30)

\subsection{Numerical results}

\subsubsection{Two risky assets}

Consider $N=2$, this is the case when there are two risky assets. For the numerical calculation, we choose $\lambda=0.1, k=0.03$ (if $k \neq 0$ ), $\alpha_{1}=0.11$, $\alpha_{2}=0.13, \sigma_{11}=0.3, \sigma_{12}=0.2, \sigma_{21}=0.2, \sigma_{22}=0.3, r=0.07, \delta=0.1, \gamma=0.3$. Then, $m_{1}, m_{2}$, and $m_{12}$ are given by $m_{1}=0.96, m_{2}=0.89, m_{12}=0.51$. As mentioned before, we discuss the cases where no borrowing or short-selling is allowed. Depending on the values of $k$ and $\lambda$, we discuss two cases of the problem (2.25) numerically and analytically in Subsections 2.5.1.1 to 2.5.1.2.

\subsubsection{The case when $k=0$.}

This is the case where there are no fixed transaction costs but there are proportional transaction costs. We first explain various notations in Figure 2.1. It is easy to see that the first octant has been divided into an outer space and an inner space by four curved planes. The outer space are the transaction regions which will be discussed in Figure 2.2 while the inner space is the No-Transaction region. In Figure 2.1, the curves $C_{1}, C_{2}, C_{3}$, and $C_{4}$ are the sets of points $(\bar{x}, \bar{y}, \bar{z})$ at which the value function attains its maximum values while trading two stocks. The optimal portfolio curve $C_{1}$ is the set of $(\bar{x}, \bar{y}, \bar{z})$ at which the value function attains its maximum by buying two stocks; curve $C_{2}$ is the set of $(\bar{x}, \bar{y}, \bar{z})$ at which the value function attains its maximum by buying the first stock but selling the second stock; curve $C_{3}$ is the set of $(\bar{x}, \bar{y}, \bar{z})$ at which the value function attains its maximum by selling the first stock but buying the second stock; curve $C_{4}$ is the set of $(\bar{x}, \bar{y}, \bar{z})$ at which the value function attains 
its maximum by selling two stocks. The curves $B_{1} \mathrm{~s}, B_{2} \mathrm{~s}, B_{3} \mathrm{~s}$ and $B_{4} \mathrm{~s}$ are the sets of points $(\bar{x}, \bar{y}, \bar{z})$ at which the value function attains its maximum values while trading only one stock out of the two stocks. The optimal portfolio curves $B_{1} \mathrm{~s}$ are the sets of $(\bar{x}, \bar{y}, \bar{z})$ at which the value function attains its maximum by buying the first stock but do not trade the second stock; curves $B_{2} \mathrm{~s}$ are the sets of $(\bar{x}, \bar{y}, \bar{z})$ at which the value function attains its maximum by selling the first stock but do not trade the second stock; curves $B_{3}$ s are the sets of $(\bar{x}, \bar{y}, \bar{z})$ at which the value function attains its maximum by do not trade the first stock but buying the second stock; curves $B_{4} \mathrm{~S}$ are the sets of $(\bar{x}, \bar{y}, \bar{z})$ at which the value function attains its maximum by do not trade the first stock but selling the second stock.

In Figure 2.2, regions $R_{1}, R_{2}, R_{3}$ and $R_{4}$ are the transaction regions of trading two stocks. For instance, if the values of our portfolio $\left(x_{1}, y_{1}, z_{1}\right)$ are in $R_{1}$, then we need to buy both of the two stocks. The optimal portfolio will be $\left(\bar{x}_{1}, \bar{y}_{1}, \bar{z}_{1}\right)$, which is one of the points on $C_{1}$. The transaction size will be determined by the distance between the optimal portfolio and original portfolio which is $\left(\bar{x}_{1}-x_{1}, \bar{y}_{1}-y_{1}, \bar{z}_{1}-z_{1}\right)$. Regions $R_{5}, R_{6}, R_{7}$ and $R_{8}$ are the transaction regions of trading only one stock. For example, if our portfolio with coordinates which is in region $R_{5}$, we need to buy the first stock but do not trade the second stock. The optimal portfolio will be one of the points on $B_{1}$ s. The transaction size will be calculated by the distance between the optimal portfolio and the original portfolio. Region $R_{1}$ is the transaction region of buying two stocks; region $R_{2}$ is the transaction region of buying the first stock and selling the second stock; region $R_{3}$ is the transaction region of selling the first stock but buying the second stock; region $R_{4}$ is the transaction region of selling two stocks; region $R_{5}$ is the transaction region of buying the first stock but do not trade the second stock; region $R_{6}$ is the transaction region of selling the first stock but do not trade the second stock; region $R_{7}$ is the transaction region of do not trade the first stock but buying the second stock; region $R_{8}$ is the transaction region of do not trade the first stock but selling the second stock.

\subsubsection{The case when $k>0$.}

This case consists of both fixed and proportional transaction costs. Similar to Figure 2.1, Figure 2.3 shows the first octant has been divided into two parts by four curved planes. The outer part are the transaction regions which will be discussed in Figure 2.4 while the inner part is the No-Transaction region. In Figure 2.3, the curves $C_{1}, C_{2}, C_{3}$, and $C_{4}$ are the sets of $(\bar{x}, \bar{y}, \bar{z})$ at which the value function attains its maximum values while trading two stocks and the curves $B_{1} \mathrm{~s}, B_{2} \mathrm{~s}, B_{3} \mathrm{~s}$ and $B_{4} \mathrm{~s}$ are the sets of $(\bar{x}, \bar{y}, \bar{z})$ at which the value function attains its maximum values while trading only one stock. In Figure 2.4, regions $R_{1}, R_{2}, R_{3}$ and $R_{4}$ are the transaction regions of trading two stocks. Regions $R_{5}, R_{6}, R_{7}$ and $R_{8}$ are the transaction regions of trading only one stock.

It is obvious to see that every two connected curved planes have intersect curves which are $C_{1}, C_{2}, C_{3}$ and $C_{4}$ shown in Figure 2.1. While in Figure 2.3, except for the parts of the curved planes bounded by the four curved planes, 


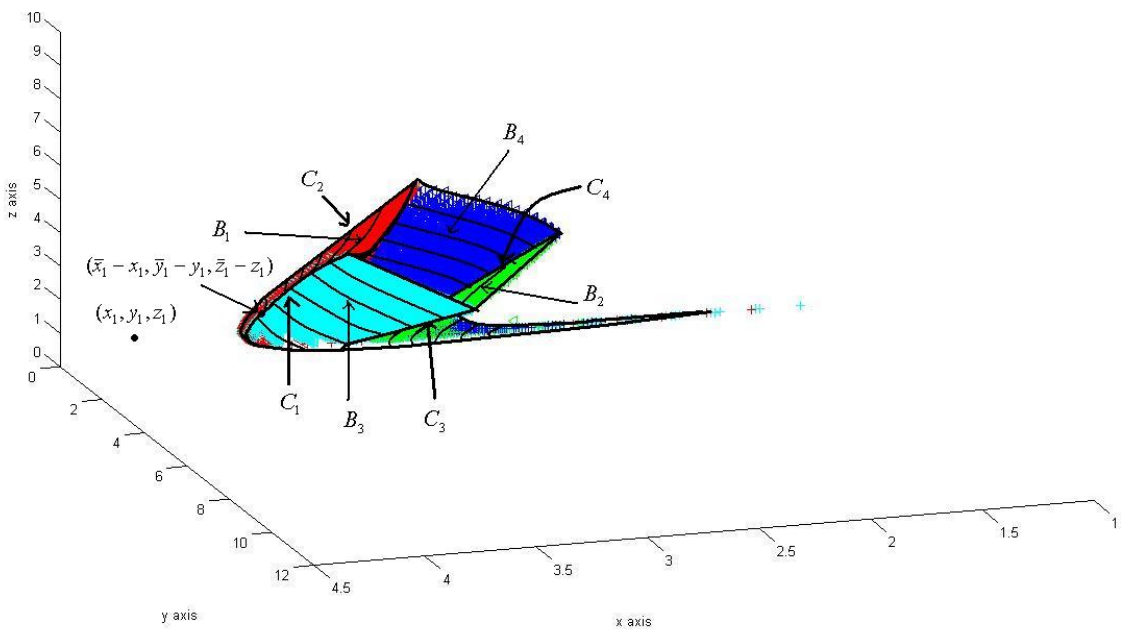

Figure 2.1: Boundaries of transaction regions and NT region when $k=0$.

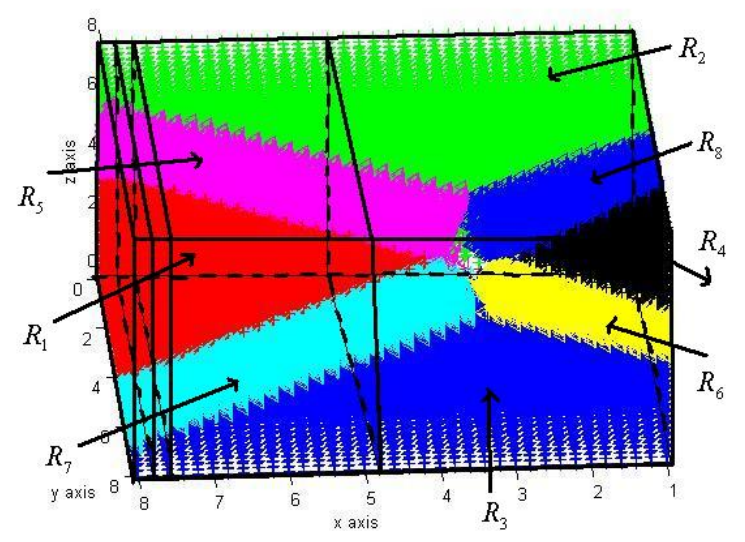

Figure 2.2: Transaction regions when $k=0$. 


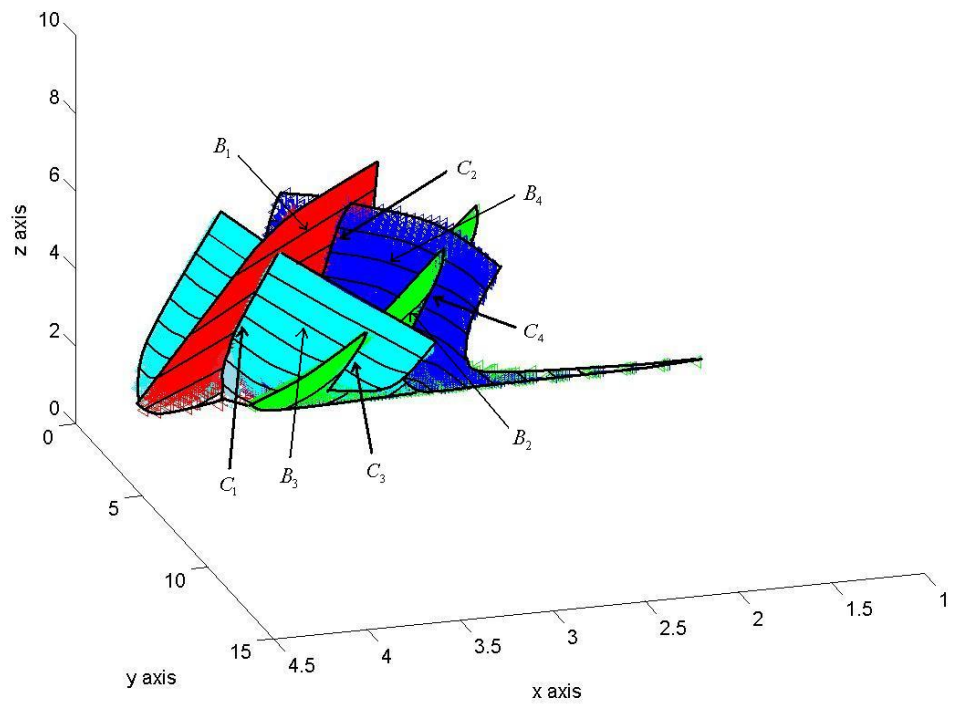

Figure 2.3: Boundaries of transaction regions and NT region when $k>0$.

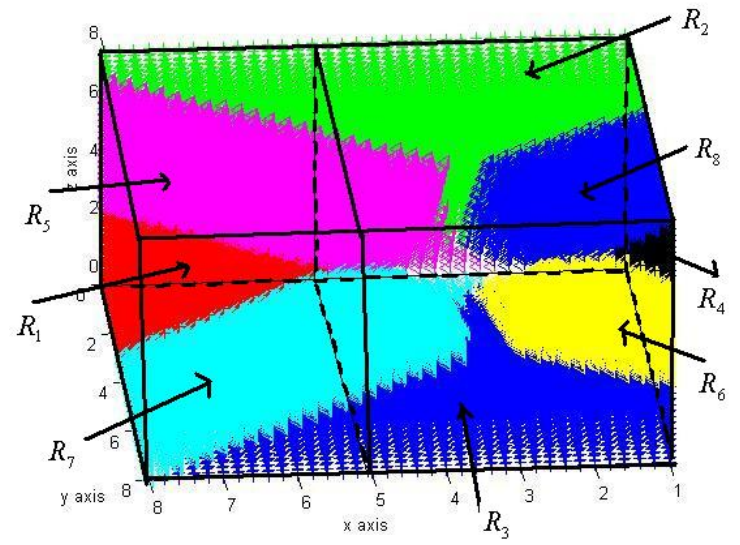

Figure 2.4: Transaction regions when $k>0$. 


\begin{tabular}{|c|c|c|c|c|c|}
\hline Ticker Name & AXP & BA & CAT & CSCO & CVX \\
\hline \hline TRT1M & 0.00743 & 0.009417 & 0.015416 & 0.00261 & 0.013074 \\
\hline \hline Ticker Name & DD & DIS & HD & HPQ & IBM \\
\hline \hline TRT1M & 0.005802 & 0.007068 & 0.004486 & 0.006387 & 0.010469 \\
\hline
\end{tabular}

Table 2.1: TRT1M

there are some extra parts of the planes exceed the intersect curves. The reason makes this happen is because of the fixed transaction cost $k$. And comparing the No-Transaction regions between the cases where $k=0$ and $k>0$, we see the No-Transaction region where $k=0$ is smaller than the No-Transaction region where $k>0$. This is easy to see from the hollow regions in Figure 2.2 and Figure 2.4

\subsection{2 $N$ risky assets}

In section 2.3, the explicit solution to equation (2.11) is given by (2.18). A simplified explicit solution which also satisfies (2.11) is given by (2.26). We use (2.26) to be our value function in this subsection. Our aim is to figure out how to trade $N$ risky assets. We study the data from ten Dow Jone Industrial Average companies. The average TRT1M (Monthly Total Return) for these ten stocks from January 2005 to December 2011 are given in Table 2.1. The symmetric variance and covariance matrix for these stocks are given in Table 2.2.

\subsubsection{Algorithm I}

The algorithm to specify all of the combinations for trading $N$ stocks is: If only one stock is traded out of $N$ stocks, there will be $C_{N}^{1}$ choices, and if exactly two stocks are traded, there will be $C_{N}^{2}$ choices. Similarly, exactly $i(i=1,2, \ldots, N)$ stocks are traded will lead to $C_{N}^{i}$ choices. Thus, there will be $C_{N}^{1}+C_{N}^{2}+\ldots+C_{N}^{N}=2^{N}-1$ combinations in total.

For the numerical calculation, we choose $\lambda=0.002, k=7, r=0.001$, $\delta=0.002, \gamma=0.3, x=2000,\left(y_{1}, y_{2}, \ldots, y_{10}\right)=(0,0, \ldots, 0)$ and by using the data from Table 2.1 and Table 2.2, we get the optimal $\xi$ values (how much we should put in each stocks) after enumerate all the $2^{10}-1$ choices in Table 2.3. The ten stocks' monthly closed prices in January 2012 are shown in Table 2.4. Table 2.3 and Table 2.4 provide us the information on how to trade the ten stocks in order to make the investment to be optimal. If we have $\$ 2000$ in our bank account and no money in the stocks accounts at the beginning, theoretically we have to buy stock AXP for $\$ 463.4$ (9.24 shares), buy stock CSCO for $\$ 807.8$ (41.12 shares), buy stock DD for $\$ 195.7$ (3.85 shares), buy stock HD for $\$ 290.5$ (6.54 shares), and buy stock HPQ for $\$ 76.9$ (2.75 shares). We will not trade stocks BA, CAT, CVX, DIS and IBM, since their $\xi$ values are either less than the fixed transaction cost which is $\$ 7$ or less than the corresponding stock prices. In the real life, we can only trade integer shares of stocks. Hence we buy AXP for 9 shares; buy 


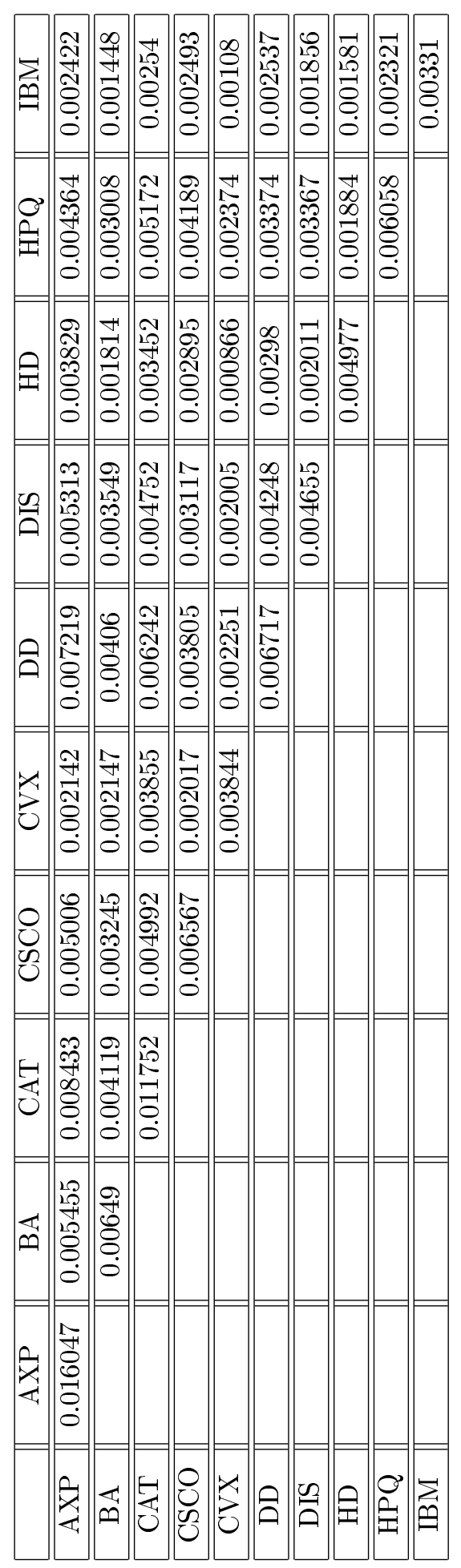

Table 2.2: Variance and Covariance matrix 


\begin{tabular}{|c|c|c|c|c|c|}
\hline Ticker Name & AXP & BA & CAT & CSCO & CVX \\
\hline \hline$\xi$ (dollars) & 463.4 & 13.14 & 7.0 & 807.8 & 4.3 \\
\hline \hline Ticker Name & DD & DIS & HD & HPQ & IBM \\
\hline \hline$\xi$ (dollars) & 195.7 & 28.8 & 290.5 & 76.9 & 6.0 \\
\hline
\end{tabular}

Table 2.3: $\xi$ values

\begin{tabular}{|c|c|c|c|c|c|}
\hline Ticker Name & AXP & BA & CAT & CSCO & CVX \\
\hline \hline Stock prices (dollars) & 50.14 & 74.18 & 109.12 & 19.65 & 103.12 \\
\hline \hline Ticker Name & DD & DIS & HD & HPQ & IBM \\
\hline \hline Stock prices (dollars) & 50.89 & 38.9 & 44.39 & 27.98 & 192.6 \\
\hline
\end{tabular}

Table 2.4: Stock prices

CSCO for 41 shares; buy DD for 3 shares; buy HD for 6 shares; and buy HPQ for 2 shares; and there are $\$(2000-7 \times 5-(1+0.002) \times 50.14 \times 9-(1+0.002) \times 19.65 \times$ $41-(1+0.002) \times 50.89 \times 3-(1+0.002) \times 44.39 \times 6-(1+0.002) \times 27.98 \times 2)=\$ 229.66$ left in the bank account. The optimal value for the value function is 10179.

Note that there are five stocks that we will not trade. Next we consider trading the rest five stocks only. We use the same parameters as we do for $N=10$, but in this case $N=5$. Let $p$ and $y$ start from $p=(0,0,0,0,0)$ and $\left(y_{1}, y_{2}, \ldots, y_{5}\right)=(0,0, \ldots, 0)$. The optimal $\xi=(485.3,852.4,202.5,310.9,76.4)$ after we enumerate all $2^{5}-1$ choices. Together with the information given in Table 2.4, the conclusion is: buy AXP, CSCO, DD, HD and HPQ for 9 shares, 43 shares, 3 shares, 7 shares and 2 shares respectively. The amount of money left in the bank account will be $\$ 145.8$. The optimal value for the value function is 10562 . We know the optimal value for the value function when trade the ten stocks is 10179, while the optimal value for the value function when trade the five stocks is 10562 . The actural difference between the two optimal values is $10562-10179=383$, and the percentage difference is $\frac{10562-10179}{10562}=3.63 \%$.

\subsubsection{Algorithm II}

The numerical results shown in subsection 2.5.2.1 are the results specifying all of the $2^{10}-1$ cases. For some large power $N$, explicit enumeration is normally impossible due to the exponentially increasing number of potential solutions. Branch and Bound method is the mostly widely used tool for solving large scale combinatorial optimization problems. The briefly idea for iteration has three main components: selection of the node to process, bound calculation, and branching. A tentative algorithm to solve our problem by using similar idea from the $\mathrm{B} \& \mathrm{~B}$ method is given below. Figure 2.5 illustrates the algorithm.

- Let $p$ be a $1 \times N$ vector with each entries are either 0 or 1 , which means we will not trade the $i$ th stock if the entry on the $i$ th position of $p$ is 0 or we will trade the $i$ th stock if the entry on the $i$ th position of $p$ is 1 . 
- Set the initial $p=(0,0, \ldots, 0)$ if we start from have not traded any one of the $N$ stocks (or set the initial $p=(1,1, \ldots, 1)$ if we start from trading all of the $N$ stocks). Denote this to be node level 0 . Calculate the value for the intervention operator

$$
\begin{aligned}
M v= & a x^{\prime \gamma}+\sum_{i=1}^{N} b_{i} y_{i}^{\prime m_{i}}+\sum_{i, j=1, i \neq j}^{N} b_{i j}\left(y_{i}^{\prime} y_{j}^{\prime}\right)^{m_{i j}} \\
= & a\left(x-k \widetilde{p}-\sum_{i=1}^{N} \xi_{i}-\lambda \sum_{i=1}^{N}\left|\xi_{i}\right|\right)^{\gamma}+\sum_{i=1}^{N} b_{i}\left(y_{i}+\xi_{i}\right)^{m_{i}} \\
& +\sum_{i, j=1, i \neq j}^{N} b_{i j}\left[\left(y_{i}+\xi_{i}\right)\left(y_{j}+\xi_{j}\right)\right]^{m_{i j}}
\end{aligned}
$$

where $\widetilde{p}$ is the sum of all $1 \mathrm{~s}$ in $p$. Store the current $M v$ value to be the incumbent value.

- Branch into two branches $p=(0,0,0, \ldots, 0)$ and $p=(1,0,0, \ldots, 0)$, and this indicates to trade the 1 st stock or not to trade it. Both of the two nodes are assumed in node level 1 (If use the initial $p=(1,1, \ldots, 1)$, branch into $p=(1,1, \ldots, 1)$ and $p=(0,1, \ldots, 1))$. Calculate the two $M v$ values. If any $M v$ value is greater than the incumbent value, keep a record for the new $M v$ and also store the corresponding $p$. For example, at node $p=(1,0,0, \ldots, 0)$ we have a greater $M v$ value. We will branch this node into two branches with nodes $p=(1,0,0, \ldots, 0)$ and $p=(1,1,0, \ldots, 0)$.

- Repeat the procedures provided in the above steps, we may get the optimal strategy on trading $N$ stocks.

If we use the algorithm illustrated above, the way to bound the calculations at each nodes will be studied in the near future. A short cut to solve the problem is: set initial $p=(1,1, \ldots, 1)$; get $\left(\xi_{1}, \xi_{2}, \ldots, \xi_{N}\right)$ by using the function 'fmincon' in Matlab; determine the maximum of $\left|\xi_{i}\right|$ happens on the $i$ th position of $\left(\xi_{1}, \xi_{2}, \ldots, \xi_{N}\right)$; make the $i$ th position of $p$ be 0 and recalculate the problem; continue doing this until we get $p=(0,0, \ldots, 0)$; among the $N$ times of calculations the optimal result has been stored. Identical result shows up if we use the same example in subsection 2.5.2.1. 


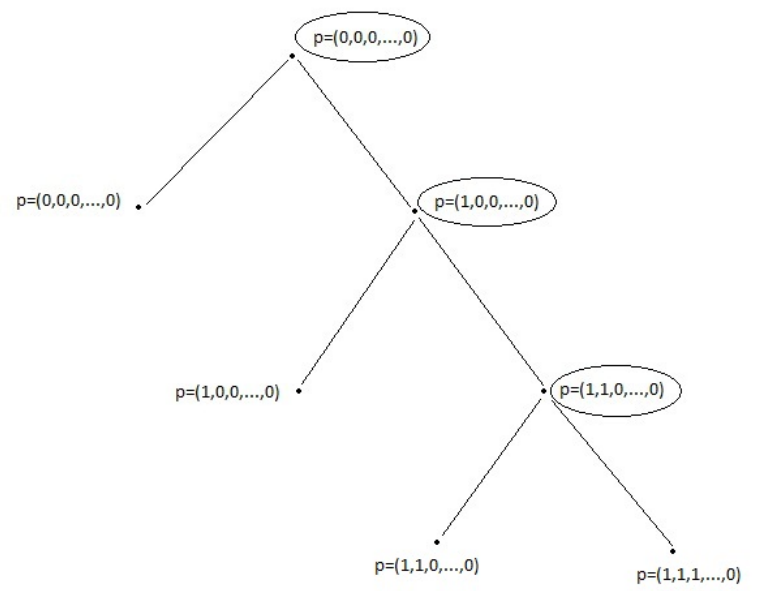

Figure 2.5: Branch and Bound graph 


\section{Bibliography}

[1] Y. Aït-Sahalia, J. Diaz and T. Hurd, Portfolio choice with jumps: a closed-form solution, Ann. Appl. Probab., 19 (2009), pp. 556-584.

[2] M. Akian, J. Menaldi, and A. Sulem, On an investmentconsumption model with transaction costs, SIAM J. Control Optim., 34 (1996), pp. 329-364.

[3] M. Akian, A. Sulem, and M. Taksar, Dynamic optimization of long term growth rate for a portfolio with transaction costs and logarithmic utility, Math. Finance., 11 (2001), pp. 153-188.

[4] C. Atkinson and P. Ingpochai, The influence of correlation on multi-asset portfolio optimization with transaction costs, J. Comput. Finance., 10 (2006/07), pp. 53-96.

[5] K. Brekke and B. Øksendal, A verification theorem for combined stochastic control and impulse control, in stochastic analysis and related topics, Progr. Probab. 42 (1998), pp. 211-220.

[6] J. Chancelier, B. Øksendal and A. Sulem, Combined stochastic control and optimal stopping, and application to numerical approximation of combined stochastic and impulse control, Proc. Steklov Inst. Math., 237 (2002), pp. 140-163.

[7] M. Crandall, H. Ishii, and P. Lions, User's guild to viscosity solutions of second order partial differential equations, Bull. Amer. Math. Soc., 27 (1992), pp. 1-69.

[8] M. Davis and A. Norman, Portfolio selection with transaction costs, Math. Oper. Res., 15 (1990), pp. 676-713.

[9] J. Eastham and K. Hastings, Optimal impulse control of portfolios, Math. Oper. Res., 13 (1988), pp. 588-605.

[10] W. Fleming and H. Soner, Controlled Markov processes and viscosity solutions, Springer, New York, 2006. 
[11] N. Framstad, B. Øksendal, A. Sulem, Optimal consumption and portfolio in a jump diffusion market with proportional transaction costs, J. Math. Econom., 35 (2001), pp. 233-257.

[12] Z. Jia, Some properties of the Hadamard product matrix, Journal of mathematics for technology., 14 (1998), pp. 150-154.

[13] R. Korn, Optimal portfolio: stochastic models for optimal investment and risk management in continuous time, World Scientific, Singapore, 1997.

[14] R. Korn, Portfolio optimization with strictly positive transaction costs and impulse control, Finance Stoch., 2 (1998), pp. 85-114.

[15] H. Liu, Optimal Consumption and Investment with Transaction Costs and Multiple Risky Assets, The Journal of Finance., 1 (2004), pp. 289-337.

[16] M. Magill and G. Constantinides, Portfolio selection with transaction costs, J. Econom. Theory., 13 (1976), pp. 245-263.

[17] R. Merton, Lifetime portfolio selection under uncertainty: the continuous-time model, Rev. Econ. Statist., 51 (1969), pp. 247257 .

[18] R. Merton, Optimum consumption and portfolio rules in a continuous-time model, J. Econom. Theory., 3 (1971), pp. 373-413.

[19] K. Muthuraman, S. Kumar, Multidimensional Portforlio Optimization with Proportional Transaction Costs, Math. Finance., 16 (2006), pp. 301-335.

[20] B. Øksendal and A. Sulem, Optimal consumption and portfolio with both fixed and proportional transaction costs, SIAM J. Control Optim., 40 (2002), pp. 1765-1790.

[21] B. Øksendal and A. Sulem, Applied stochastic control of jump diffusions, Springer, 2005.

[22] S. Shreve, Stochastic calculus for finance II, continuous-time models, Springer, New York, 2004.

[23] S. Shreve and H. Soner, Optimal investment and consumption with transaction costs, Ann. Appl. Probab., 4 (1994), pp. 609-692.

[24] S. Shreve, H. Soner and G. Xu, Optimal investment and consumption with two bonds and transaction costs, Math. Finance., 1 (1991), pp. 53-84. 